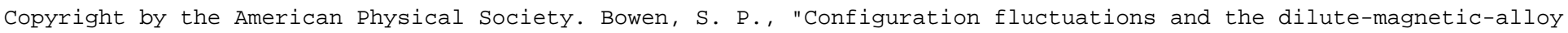
problem," Phys. Rev. B 18, 3400 DOI: http://dx.doi.org/10.1103/PhysRevB.18.3400

\title{
Configuration fluctuations and the dilute-magnetic-alloy problem
}

\author{
Samuel P. Bowen \\ Department of Physics, Virginia Polytechnic Institute and, State University, Blacksburg, Virginia 24061
}

(Received 5 January 1977)

\begin{abstract}
A new theoretical treatment of the Anderson model of transition-metal or rare-earth impurities in a simple metal is presented. This study treats the impurity Coulomb correlation exactly and the host impurity coupling to lowest order in the sense of a self-consistent degenerate perturbation theory. The central result of this work is that if an impurity interconfigurational excitation energy (ICEE) is close to the Fermi energy in the atomic limit, a temperature-dependent shift of the ICEE is found for the interacting system. This temperature-dependent shift is shown to give a good description of the experimental observations on dilute magnetic alloys.
\end{abstract}

\section{INTRODUCTION}

For many years the problem of transition-metal or rare-earth impurities in noble metal hosts has been studied theoretically be examining the simpler $S$-shell Anderson model. ${ }^{1}$ This model Hamiltonian has a noninteracting part $H_{0}$ representing the single impurity atom states and the conduction electrons of the host. In the usual notation $^{2} H_{0}$ is written as

$$
H_{0}=\sum_{s} \epsilon_{d s} n_{d s}+\frac{1}{2} \sum_{s} U n_{d s} n_{d \vec{s}}+\sum_{\overrightarrow{\mathrm{k}} s} \epsilon_{\overrightarrow{\mathrm{k}} s} n_{\overrightarrow{\mathrm{k}} s} .
$$

Within this Anderson model, the impurity and host conduction electron systems are coupled together by a one-electron hopping term $V$ which represents a conduction electron hopping on and off the impurity site

$$
V=\sum_{\overrightarrow{\mathrm{k} s}} V_{\overrightarrow{\mathrm{k}}_{d}}\left(c_{d s}^{\dagger} c_{\overrightarrow{\mathrm{k} s}}+c_{\mathrm{k} s}^{\dagger} c_{d s}\right) .
$$

In many applications of this model the coupling matrix elements $V$ are regarded as small. Though this is not essential to much of what follows, the major thrust of this work will assume a small $V_{k d}$.

The major thrust of this study, besides assuming a small $V_{k d}$, is to treat the impurity correlation and many-electron configurations exactly, and to examine under what circumstances such a system could reproduce the experimental properties of dilute magnetic alloy systems. The consequences of this study form a new theory of the dilute alloy which is a significant departure from previous theoretical descriptions. Because the results are so different it is well to briefly summarize the main points, even though heuristic and detailed presentations are also given below.

First, the method used here is rooted in the ionic picture of Schrieffer ${ }^{2}$ and Hirst $^{3}$ (SH). Products of many-electron impurity states and host conduction-band states are taken as initial states for the alloy system. The equations of motion of the stepping operator $\mathrm{s}^{4}$ between many-electron states which differ by one electron are studied in a formalism which combines elements of Feenberg perturbation theory, ${ }^{5}$ evaluation of truncated secular equations as in degenerate perturbation theory, and a self-consistent determination of thermodynamic correlation functions. ${ }^{6}$

Second, the strongest effect of the impurity-host coupling is found in what the "Kondo" picture? would describe as a strong-coupling regime, and which in this picture is best described as the near degeneracy of the unperturbed states of the alloy system. To be more explicit the strongest effect of the $V_{k d}$ coupling is found when the energies of an $n$ electron impurity configuration and the $\mathrm{N}$ electron host Fermi sea are approximately degenerate with $n \pm 1$ electron-impurity configuration and the $N \mp 1$ electron-Fermi sea of the host. That a small matrix element should show its greatest effect when the unperturbed states are degenerate is well known. However, in this context the conditions manifesting a many-electron degeneracy have not been considered before. As is shown below in an heuristic discussion the alloy many-electron degeneracy implies that the impurity configuration energies $E_{n}$ and $E_{n+1}$ are not degenerate but differ by an energy equal to the conduction-electron chemical potential $\mu$, that is,

$$
E_{n+1}-E_{n} \approx \mu \text { or } E_{n}-E_{n-1} \approx \mu
$$

The physical relevance of this condition rests on two consequences of this study. First, the unperturbed energy $E_{n+1}-E_{n}-\mu$ occurs as one of the atomic limit interconfigurational excitation energies (ICEE's) of impurity Green's function when the host and impurity are in equilibrium. Second, our calculation for the coupled system derives a termperative-dependent energy shift $\delta E_{n}$ for these poles of the impurity Green's 
function in thermodynamic equilibrium. The temperature dependence of this shift in the interconfigurational citation energy (ICEE), $E_{n+1}-E_{n}$ $-\mu-\delta E_{n}$ is shown in this paper to give a fairly good description of the experimental dilute alloy effects.

The departure from the traditional perspective of the Friedel-Anderson ${ }^{8}$ or Kondo ${ }^{9}$ approaches to this problem is apparent when one identifies the interconfigurational energies of the Anderson model $\epsilon_{d s}-\mu$ or $\epsilon_{d s}+U-\mu$. The considerations described above lead us to examine the consequences of one of these energy differences being close to zero. In the traditional approaches to this problem, both of these energies are considered as being large in absolute value and of opposite sign. In this work a "magnetic" response is obtained for $\epsilon_{d s}-\mu \leqslant 0$ and $\epsilon_{d s}-\mu+U \geqslant 0$ as for previous studies, but the parameter values for the model are quite different. The essential difference between our perspective and previous application using the Anderson-model Hamiltonian is that we regard the model parameters as arising from an ionic context, while previous treatments have used Hartree-Fock (HF) calculations to determine $\epsilon_{d s}$ and $U$. As discussed in Appendix D, these HF estimates tend to emphasize the one electron energies far from the Fermi energy. It is an interesting commentary on the tendency of HF estimates to be too large that the experimentally estimated lifteime broadening ${ }^{10}$ of the $d$ orbitals

$$
\Delta=\pi N(0)\left|V_{\vec{k} d}\right|^{2},
$$

where $N(0)$ is the density of conduction-electron states at the Fermi energy, has been consistently smaller than the initial HF calculations predicted. ${ }^{11}$ The most desirable procedure for the present work would be to evaluate the model Hamiltonian parameters using a many-electron calculation process of the type suggested by Hirst. ${ }^{12}$

The a priori evaluation of model parameters in the ionic context for even a simple Anderson model or for any more realistic model representing an iron group or rare-earth impurity is quite beyond the scope of the present effort. The aim here is to explore the consequences of this new perspective, examine what model parameter values are needed to reproduce experiment, and raise the observation that a previously unexplored constellation of hypotheses seems to explain many facets of the dilute alloy problem. The fact that my method begins by treating the many-electron effects of the impurity and represents a new and, possibly, powerful theoretical tool for such problems lends merit to the possibility that this description of the dilute alloy problem may be phys- ically relevant.

The work done in this paper differs from previous theoretical treatments in that the Anderson ${ }^{13}$ HF criterion for the presence of a magnetic moment is definitely not used. While Anderson's treatment has been criticized by many ${ }^{14}$ on the grounds that it lacks rotational invariance and does not hold in the $\Delta \rightarrow 0$ limit, it still retains a strong following, especially in the experimental literature. The criterion for magnetic behavior to be developed in this paper is simply a question of whether the impurity is weakly enough coupled to the Fermi sea to be able to independently orient its spin or whether it is strongly correlated with the rest of the conduction electrons.

The results of this paper also differ markedly from the large body of literature ${ }^{15}$ which is based on the "Kondo" themes of the impurity inducing a many-body correlated state in the host conductionelectron gas. In particular, the widely held idea of the Kondo effect being caused by a narrow resonance at the Fermi energy which is not an impurity interconfigurational excitation energy is rejected. Rather, the perspective of this work is that the ICEE peaks are not necesarily wide in energy, that estimates of the widths of the $\epsilon_{d}$ and $\epsilon_{d}+U$ peaks in the $S$-shell model have been derived througn interpretations of the model in a HF sense and could be too large. The perspective of this paper attempts to explore the proposition that the dilute magnetic alloy behavior is caused by manyelectron effects localized on the impurity site and not spread out through a cloud of surrounding conduction electrons. This picture of the magnetic behavior of the ion being localized is quite consistent with the experimental results of neutron scattering and other microscopic probes. ${ }^{16}$

Because of the recent application of Wilson's ${ }^{17}$ Kondo calculations to this Hamiltonian, ${ }^{18}$ a brief comment of comparison is in order. The theoretical methods used here are complimentary to those used by Wilson et al., though a direct comparison may be difficult because of the necessary approximations required in this work. Where the Wilson et al. method constructs explicit manyelectron states for truncated many-electron Hamiltonians, the method of this paper examines stepping operators which step between many-electron states whose electron counts differ by one. Where the Wilson method starts with small numbers of electrons in the truncated system and builds iteratively to larger number of electrons, the approach of this paper attempts to evaluate the thermodynamic expectation values of stepping operators in the thermodynamically most probable many-electron states. Of necessity this evaluation can only be determined approximately and the ap- 
proximations must be carefully examined. However, both calculations for the susceptiblity agree quite closely in the high- $T$ and low- $T$ regimes. In the intermediate temperature ranges, the two methods are not in agreement. The root of this disagreement is examined elsewhere, but one point bears discussion here. There is only one part of the Wilson procedure that is not justified in a rigorous fashion. The process of calculating with a sequence of truncated Hamiltonians has been placed on sound footing in another context by the studies of Masson. ${ }^{19}$ The numerical stability of the convergence for many-electron states with $\sim 70$ electrons is well demonstrated by the careful numerical studies of both Wilson and Krishnamurti. Wilson's a priori anzatz which replaces the uniformly dense unperturbed conduction-electron energies $\epsilon_{\overrightarrow{\mathrm{k}}} \approx\left(|\overrightarrow{\mathrm{k}}|-\left|\overrightarrow{\mathrm{k}}_{F}\right|\right) \nu_{F}$ with the discretized $\epsilon_{n} \approx \pm 1 / \Lambda^{n}$ sequence where $\Lambda=1.5$, 2, 3 underlies the whole Wilson method and has not been placed on the same solid foundation as the other elements of their calculations. The Wilson discretization anzatz replaces host conduction-electron energies near the Fermi energy, which in most models of pure system display an almost constant density of states near $\epsilon_{F}$, with a host density of states that is logarithmically singular at $\epsilon_{F}$. While such a discretization enables the application of the powerful renormalization group methods to this problem it must still be regarded as an hypothesis motivated by the wealth of perturbative studies on this problem.

A test of the Wilson discretization anzatz is well beyond the scope of this study. It may well be that such a test is not possible in the context of the current approach of this study. However, a comparison of the two methods will be made elsewhere. This discussion has been made only to indicate that in principle the theoretical approach of this study is not in contradiction with the general schema represented by the Wilson et al. calculations and that at least one element of Wilson's method is open to question. The differences between this paper and previous studies lie in approximations and hypotheses the nature of which leave open the question of the microscopic description of the dilute alloy problem. It is in this context that the following study is presented.

Further analysis and possibly new experimentation may finally resolve the differences between this approach and previous studies. In the meantime, the consequences of the ionic picture of Schrieffer and Hirst (SH) and the many-electron degeneracy of the alloy will be presented below.

To gain a more heuristic understanding for the physical picture used here, we need to adopt a general perspective. The problem we examine involves the coupling of states of a "free" Hamiltonian $H_{0}$ by a relatively small perturbation $V$. Let $\left|\psi_{A}\right\rangle$ and $\left|\psi_{B}\right\rangle$ represent two states of $H_{0}$ which will be specified more precisely below. The general consideration of Hamiltonian secular matrices suggests that a small perturbation can quite significantly alter the spectrum of $H_{0}$ if the unperturbed states are close to being degenerate in energy. ${ }^{20}$ Let us examine under what conditions for this Anderson model the small coupling $V$ could be especially important in this sense. In other words, we seek to examine the states coupled by $V$ to see under what circumstances they could be close in energy. One of the two types of states which are coupled by the potential $V$ is

$$
\left|\psi_{A}\right\rangle=\left|\psi_{F}\left(N_{F}\right)\right\rangle\left|\psi_{I}(N)\right\rangle \text {, }
$$

where $\left|\psi_{F}\left(N_{F}\right)\right\rangle$ is an $N_{F}$-electron state of the host conduction electrons, and $\left|\psi_{I}(N)\right\rangle$ is an $N$-electron impurity state. In a realistic model $\left|\psi_{I}(N)\right\rangle$ might represent a $d^{N}$ of $f^{N}$ configuration of the $d$ or $f$ shell of the impurity. The second state is

$$
\left|\psi_{B}\right\rangle=\left|\psi_{F}^{\prime}\left(N_{F}-1\right)\right\rangle\left|\psi_{I}(N+1)\right\rangle \text {. }
$$

Note first that both of these composite states correspond to having $N_{F}+N$ electrons. The energies of these two states are

$$
\begin{aligned}
& E_{A}=U_{F}\left(N_{F}\right)+E_{N}, \\
& E_{B}=U_{F}^{\prime}\left(N_{F}-1\right)+E_{N+1} .
\end{aligned}
$$

We thus seek to explore the consequences of the condition that $E_{A} \approx E_{B}$, i.e., that

$$
E_{N+1}-E_{N}-\left[U_{F}\left(N_{F}\right)-U_{F}^{\prime}\left(N_{F}-1\right)\right] \approx 0 .
$$

There are actually quite a large number of different states $\left|\psi_{F}\left(N_{F}\right)\right\rangle$ and correspondingly many different types of composite states $\left|\psi_{A}\right\rangle$ and $\left|\psi_{B}\right\rangle$ for the same impurity configurations. This makes the evaluation of (1.7) somewhat difficult, unless we adopt a thermodynamic perspective which substitutes the thermodynamically most probable state energies $U_{F}\left(N_{F}\right)$ and $U_{F}\left(N_{F}-1\right)$ into this expression. In this case, the difference

$$
U_{F}\left(N_{F}\right)-U_{F}^{\prime}\left(N_{F}-1\right) \approx \mu \approx \epsilon_{F},
$$

where $\mu$ is the chemical potential of the host conduction electrons. This reduces the condition for the approximate degeneracy of the composite states to the statement that the impurity interconfigurational excitation energy (ICEE) should be close to zero,

$$
E_{N+1}-E_{N}-\mu \approx 0 \text {. }
$$

This result says that the energies of states in two adjacent configurations of the impurity shell should have a difference which is on the order of the chemical potential of the host conduction elec- 
trons. This could mean, for example, that the two impurity configurations might differ in energy by something on the order of 5-8 eV. A priori verification of whether any atoms satisfy this condition is difficult because the configuration energies are those appropriate to the impurity inside the host metal and not to the free-atom energy differences. An impurity atom which could reasonably be a candidate for this prescription is $\mathrm{Mn},{ }^{21}$ based on its $d^{n} s^{1} \rightarrow d^{n-1} s^{2}$ core to valence transitions.

It is important at this point to emphasize that even though the preceding paragraphs have been written in the language of the conduction electrons interacting with the $d$ or $f$ shell, we would argue in agreement with Flynn ${ }^{22}$ that the impurity cell is almost always netural and that the interconfigurational excitation energies (ICEE) which we discuss are similar in magnitude to the core-valence transitions discussed by Flynn and correspond in the neutral atom to $d^{n-1} s^{2} \rightarrow d^{n} s^{1}$ transitions and others.

A second important consequence of (1.9) becomes immediately clear when it is realized that this small quantity $E_{N+1}-E_{N}-\mu$ is exactly one of the ICEE's at which the atomic limit impurity Green's function has its poles. ${ }^{23}$ Thus, the near degeneracy of composite states in the host + impurity system can give rise to ICEE resonances in the $t$ matrix which are near the Fermi energy. It is worth noting that this degeneracy of composite states in the alloy requires the close matching of two physically distinct quantities: the energy difference between impurity configurations and the host Fermi energy. The mismatch of these quantities will be seen below to give a significant depression of the effects of the coupling $V$ on the composite system.

The rest of this paper will pursue the logical consequences of the heuristic picture presented above. Our analysis employs impurity parameter values which in the usual Kondo picture would be regarded as strong $J$ coupling, i.e., large $N(0) J_{\text {eff }}=(\Delta / \pi)\left[\epsilon_{d s}^{-1}-\left(\epsilon_{d s}+U\right)^{-1}\right]$.

There are at least two distinct ways in which this parameter regime can be realized. The first of these is by taking the large $V_{\overrightarrow{\mathbf{k}} d}$ limit, in which the $d$-lifetime broadening $\Delta$ dominates all other parameters in the problem. In this case the temperature behavior of thermodynamic quantities like the susceptibility is determined by comparing the temperature $T$ with $T_{\Delta}=\Delta / \pi k_{B}$, where $k_{B}$ is the Boltzmann constant. For temperature below $T_{\Delta}$ we have a constant Pauli-like susceptibility

$$
\chi=\frac{\mu_{B}^{2}}{\pi \Delta}\left[1+\frac{U}{\pi \Delta}+O\left(\left(\frac{U}{\Delta}\right)^{2}\right)\right] .
$$

This is not the regime that we will study here, for as is well-known in such a regime it makes little sense to begin with an ionic or atomic picture of the impurity.

The other strong $J$-coupling situation corresponds to the degeneracy of two composite states of the alloy and in the $S$-shell model to either $\epsilon_{d s}$ or $\epsilon_{d s}+U$ being small [cf. Eq. (1.6)]. For this model $\epsilon_{d s}$ is the energy difference between $N=1$ and $N=0$ configurations, while $\epsilon_{d s}+U$ is the energy difference between $N=2$ and $N=1$ configurations. Both of these quantities are measured with respect to the Fermi energy and so do not explicitly display the chemical potential. ${ }^{24}$

The degeneracy of composite states of the system and the resulting placement of an ICEE near the Fermi energy implies that we must encounter statistical fluctutations in the grand canonical ensemble between the impurity configurations connected by the ICEE. The work reported below displays the connection between the configuration fluctuation phenomena ${ }^{25}$ and the dilute magnetic alloy phenomena.

This paper consists of five sections. Section II will briefly review the ionic approach of Hirst and Schrieffer (SH). Section III will lay the theoretical frame work for doing degenerate perturbation theory in the grand canonical ensemble. In particular, we examine here the atomic limit, display the relationship to the Hartree-Fock approximation (HFA), and examine the effects of the coupling on the impurity states. In Sec. IV, we describe the main theoretical calculations underlying this report and display their application to a few experimental properties. Many of the theoretical details are relegated to appendices $A, B, C$, and $D$, hopefully to enhance the readability of our results. Finally, in Sec. V, the extension of these ideas to realistic many-electron atoms and to other problems is examined.

\section{THE IONIC APPROACH AND THE ICEE'S}

Hirst $t^{26}$ in his important work outlined a description of transition or rare earth ions in metals which incorporated the earlier ionic-based perspective of Schrieffer. ${ }^{27}$ Hirst observed that when the impurity potential is strong enough to give a fairly narrow virtual bound state (VBS) at the impurity site, the traditional one-electron picture is no longer valid and a correlated manyelectron basis becomes important. This occurs because the strong impurity potential and the narrowness of the VBS implies that the electrons spend more time in the impurity, thus enhancing the importance of the local-local Coulomb interactions and the correlation that they imply. It is 
this sort of argument which led Hirst to begin the description of the dilute alloy with the impurity atomic states in the zeroth approximation.

The principle accomplishment of Hirst's approach was to show that by starting with the essentially atomic point of view it is possible to derive from first principles a Hamiltonian with three parts,

$$
H=H_{\text {ion }}+H_{\text {cond }}+H_{\mathrm{I}},
$$

where $H_{\text {ion }}$ represents in zeroth order the ion Hamiltonian which contains Coulomb interactions between the electrons, spin-orbit interactions, and interactions with the impurity nucleus, the closed-shell electrons, the lattice ions and the conduction electrons in their lowest states. This essentially atomic Hamiltonian has correlated many-electron states $\Psi_{i}^{(n)}(\alpha)$ which contain $n$ electrons in the $d(\operatorname{or} f)$ shell and have term energies $E_{n}(\alpha)$. Here $\alpha$ represents the irreducible represenation to which the many-electron state $\Psi^{(n)}(\alpha)$ belongs. Where necessary; the rows of the irreducible representation will be labeled by the index $i$. In a second quantized notation the creation operators for the correlated many-electron states will be written as $b_{n}^{\dagger}(\alpha)$. These operators are $n$th order polynomials of the $d$ (or $f$ ) shell creation operators $c_{m s}^{\dagger}$. The form of the polynomials can be determined by standard methods. ${ }^{28}$

The conduction-electron states and other closedshell electron states are determined by a constrained restricted Hartree-Fock (CRHF) procedure described in detail by Hirst. ${ }^{29}$ Physically, this CRHF procedure determines one-electron orbitals for those electrons which are orthogonal to the localized shell states and which minimize the ground-state energy. The conduction-electron states are thus not only separated from the impurity states, but also contribute to the impurity screening. Far away from the impurity, these conduction-electron states are little changed from the host states. Near the impurity the conductionelectron states are more strongly altered.

In the CRHF calculation the conduction electrons are treated as independent quasiparticles and the variational CRHF calculation determines an effective interaction between the distorted conduction electrons and the $d$ (or $f$ ) shell impurity electrons. If we represent the conduction-electron annihiation operators as $c_{\overrightarrow{\mathrm{k}}_{s}}$ and the $d$-shell orbitals by $c_{d s}$, then the equation of motion for $c_{\overrightarrow{\mathrm{k}} s}$ which is derived from the CRHF variation can be written $a^{30}$

$$
\left[c_{\overrightarrow{\mathrm{k}} s}, \mathfrak{H}\right]=\epsilon_{\overrightarrow{\mathrm{k}} s} c_{\overrightarrow{\mathrm{k}} s}+\sum_{d} V_{\overrightarrow{\mathrm{k}} d} c_{d s} .
$$

In this Eq. (2.1) both the $\epsilon_{\overrightarrow{\mathrm{k}} s}$ and the $V_{\overrightarrow{\mathrm{k}} d}$ arise as variational parameters of the CRHF procedure. This equation exhibits the conduction-electron band energies $\epsilon_{\overrightarrow{\mathrm{k} s}}$ and the effective interaction matrix element $V_{\overrightarrow{\mathrm{k}} d}$ between the conduction electrons and the $d$ (or $f$ ) shell orbitals. This already specifies the form of $\mathfrak{H}_{I}$ which arises between the $d$ shell orbitals and the conduction electrons.

While Hirst did not explicitly emphasize the magnitude of $V_{\overrightarrow{\mathrm{k}} d}$ which arises in his CRHF method, it must be quite small for his analysis to be valid. In particular, Hirst limited his discussion to firstorder perturbation theory, neglecting the lifetime broadening of the atomic states. Hirst argued that this was permissible if the minimum interconfigurational excitation energy $E_{\text {exc }}$,

$$
E_{\text {exc }}=\min \left|E_{N}(\alpha)-E_{N \pm 1}(\beta)\right|,
$$

was relatively large. Hirst estimated that $E_{\text {exc }}$ should be roughly on the order of the Slater $F^{0}$ integrals and thus might be expected to be as large as $25 \mathrm{~V}$. This estimate neglects one of the important characteristics of the CRHF calculation, namely, that it includes the screening of the $d$ states by the conduction electrons in zeroth order. While it is impossible to evaluate $E_{\text {exc }}$ unequivocally, short of doing a CRHF calculation itself, we can gain insight into the allowed range of values by reviewing the appropriate estimates by Herring $^{31}$ for $E_{\text {exc }}$. It may be recalled that Herring estimated from comparison with atomic spectra and other estimates of theories of conductionelectron screening that the "effective" Slater integral for the $3 d$ shell could be in the range $4-6$ eV. This range of values was obtained essentially by examining energy differences between $3 d^{n} 4 s^{1}$ and $3 d^{n-1} 4 s^{2}$ configurations instead of energy differences within a pure $d$ shell alone.

Our interest in this paper is to look at Hirst's model in the grand canonical ensemble where the corresponding version of $E_{\text {exc }}=\min \mid E_{n}(\alpha)$ $-E_{n \pm 1}(\beta) \mp \mu \mid$ is small and to do degenerate perturbation theory using essentially secular equations or Feenberg techniques. ${ }^{32}$

At this stage it is appropriate to make again a brief comparison between the Schrieffer-Hirst ionic approach and the traditional Friedel-Anderson (FA) perspective. In one sense these two pictures simply represent alternative bases from which to expand the states of the impurity and host. The FA approach tends to emphasize the itinerant aspect, but typically fails to treat carefully the inherent correlation effects because of a reliance on Hartree-Fock approximations for the local states. As Wohlleben and Coles ${ }^{33}$. have noted such an approach has led generally to theoretical results of questionable applicability to existing systems. Presumably, this itinerate approach could be made to properly describe these systems, but it seems to require very complicated 
linear combinations of states to diagnolize the Hamiltonian.

The SH approach, on the other hand, begins in a representation which already recovers the proper atomic limitas $V_{\mathrm{k} d} \rightarrow 0$. As noted by many researchers ${ }^{34}$ the FA approach fails to recover this limit at all. As will be shown below the SH approach must be studied in a fashion that includes the lifetime broadening of the impurity states, especially if the impurity ICEE is degenerate with a quasicontinuum of host conduction-electron states.

The first task is to consider what kinds of energies and operators associated with the correlated states are actually involved in the interaction (2.1) For an understanding of the resistivity, for example, it is necessary to determine the $t$-matrix for scattering from the impurity. As is wellknown, ${ }^{35}$ this is proportional to the one-electron impurity Green's function. This means that the $t$-matrix energies will be the same as those which occur as poles in the impurity one-electron Green's function.

While the exact nature of the ICEE poles of the one-electron Green's function for many-electron systems has been known for several years, many workers continue to compare conduction-electron single-particle energies with many-electron atomic-configuration energies as well as with the HF Koopman's energies. As emphasized by Hubbard ${ }^{36}$ the excitation energies of the one-electron Green's function correspond to the energy at which electrons bearing certain single-particle quantum numbers can be added to the system. In particular, then the "one-electron" energies should correspond to all possible ICEE differences

$$
\epsilon_{n}(\alpha, \beta)=E_{n}(\beta)-E_{n-1}(\alpha),
$$

between configurations of the impurity system which differ by one electron. The irreducible representations of the many-electron states $\Gamma_{\beta}^{(n)}$ and $\Gamma_{\alpha}^{(n-1)}$ must be related to each other in such a way that the direct product of the many-electron representations, $\Gamma_{\beta}^{(n)} \times \Gamma_{\alpha}^{(n-1)}$, must contain the one-electron representation.

In general, one must expect that for given oneelectron quantum numbers $\left\{n, l, m_{l}, m_{s}\right\}$, there will be many resonances of differing weights appearing in the Green's function. A particular one-electron orbital wave function could be part of several different many-electron states: In what follows, we examine explicitly those cases in which at least two impurity configurations will be competing for population in the grand canonical ensemble.

For purposes of calculation, it will be convenient to have operators that correspond to the en- ergy differences (2.2). Physically, we need $\phi_{n}^{\dagger}(\alpha, \beta)$ operators which annihilate the $(n-1)$ electron states and replace them with the $n$-electron states. If we consider only the impurity Hamiltonian it is straightforward to show that the operator corresponding to an interconfigurational excitation (2.2) is

$$
\phi_{n}(\alpha, \beta) \approx b_{n-1}^{\dagger}(\alpha) b_{n}(\beta) .
$$

It is the perspective of our ionic based approach that if we calculate the properties of the system using as basis vectors the conduction electrons $c_{\overrightarrow{\mathrm{k}} s}^{\dagger}$, and the interconf iguration excitation operators (2.3), then we have an almost diagonal "Hamiltonian" for small coupling. The success in carrying out this program relies on a method which utilizes the above operators as "states" and reduces calculations of thermodynamic Green's functions to considerations of secular equations, resolvents, and approximation procedures like those of Feenberg. ${ }^{37}$ It is to a brief discussion of this method that the next two sections are devoted.

\section{PROPERTIES OF THE GREEN'S FUNCTION AND THE ATOMIC LIMIT}

In this section, we examine properties of the one-electron impurity Green's function with special emphasis on the atomic limit. Also, the basic properties of the theoretical technique which we use in this paper will be developed in this section. The essential criteria for the development of the scheme are that it should start with a proper treatment of atomic correlation, that exact properties of the Green's function be reproduced, and that any scheme reproduce the atomic limit as the coupling $V_{\overrightarrow{\mathrm{k}} d}$ goes zero.

First, we examine the exact properties of the one-electron impurity Green's function. We examine here the retarded Green's function for the impurity one-electron orbital $c_{d s}$ which may be written as

$$
G_{d s}(t)=-i\left\langle\left\{c_{d s}(t), c_{d s}^{\dagger}(0)\right\}\right\rangle \Theta(t) .
$$

Here $\langle\cdots\rangle$ reflects the thermal average. $\{$,$\} is$ an anticommutator, and $\Theta(t)$ is a unit-step function. ${ }^{38}$

Hubbard $^{39}$ has analyzed this Green's function in the atomic limit and has detailed its exact properties. The most important of these characterizes the spectrum of the Fourier transform $G_{d s}(\omega)$. Following Zubarev, ${ }^{40}$ if $|p\rangle$ and $|q\rangle$ represent exact eigenstates of the Hamiltonian $H-\mu N$, which have different numbers of electrons, then the Green's function $G_{d s}(\omega)$ may be written 


$$
G_{d s}(\omega)=\frac{1}{a} \sum_{p q} \frac{\left|\left\langle p\left|c_{d s}\right| q\right\rangle\right|^{2}}{\omega-\left(E_{p}-E_{o}\right)}\left(e^{-\beta E_{p}}-e^{-\beta E_{q}}\right) .
$$

Here $E_{p}, E_{q}$ are the eigenvalues of the states $|p\rangle$ and $|q\rangle$, respectively, $\beta$ is $\left(k_{B} T\right)^{-1}$ and $z$ is the grand canonical partition function for the system.

Hubbard, Zubarev, and many others have analyzed the calculation of thermodynamic Green's functions from many points of view. These have included straightforward evaluation if the eigenstates are known, truncation and approximate solution of the hierarchy of equations of motion, and the standard perturbation expansions of quantum field theory.

The theoretical methods of this paper rely on an extension and generalization ${ }^{41}$ of the ideas of Lonke $^{42}$ and Feenberg. ${ }^{43}$ This generalization places the calculation of one-electron Green's functions into the context of standard matrix theory, but on a space whose vectors are second-quantized operators and whose scalar product is the grand canonical ensemble average of the anticommutator of second-quantized operators.

The essential ideas are the following: instead of working with the Hamiltonian $H-\mu N$ and directly seeking its eigenvectors and eigenvalues, it proves to be slightly more convenient to work with the $L$ operator which is defined as the commutator with $H-\mu N,{ }^{44}$

$$
L \alpha=[\alpha, H-\mu N],
$$

where $\alpha$ is an operator. The time dependence of an operator $\theta$ is given by $(\hbar=1)$

$$
\mathcal{O}(t)=e^{-i L t} \mathcal{O}(0) \text {. }
$$

The Fourier transformed Green's function $G_{d s}(\omega)$ may then be written as

$$
G_{d s}(\omega)=\left\langle\left\{(\omega+i \delta-L)^{-1} c_{d s}, c_{d s}^{\dagger}\right\}\right\rangle,
$$

where $\delta=0^{+}$and $\omega$ is real. This expression may be written in a more transparent form if it is recognized that a scalar product between operators $A$ and $B$ can be defined for a Hilbert space of second-quantized Fermion operators, ${ }^{45}$

$$
((A, B))=\left\langle\left\{B, A^{\dagger}\right\}\right\rangle \text {. }
$$

This scalar product shows the one-electron Green's function to be a diagonal matrix element of a resolvent $(\omega-L)^{-1}$ of a symmetric operator $L$ :

$$
G_{d s}(\omega)=\left(\left(c_{d s},(\omega-L)^{-1} c_{d s}\right)\right) .
$$

If we were dealing with finite dimensional or diagonal representations for $L$, the determination of the various Green's functions which are involved in the theory would require knowledge of the secular matrix

$$
S_{A B}=((A,(\omega-L) B)),
$$

for a convenient orthonormal set of basis vectors. This sort of calculation is familar from standard quantum-mechanics calculations and band-structure studies in solids. The only essential difference here is that the vectors are certain second-quantized operators, and the matrix elements of $L$ will be temperature dependent. This temperature dependence simply reflects the thermal equilibrium of our system and will be the source of an ICEE shift with temperature which is one of the main results of this paper. The evaluation of the temperature dependence of the matrix elements would be trivial if we had all of the information required to accomplish the evaluation in representation (3.2). This is of course not the case, and the evaluation of the matrix elements will be obtained through the solution of thermodynamic consistency equations which express the property of detailed balance in thermal equilibrium.

In order to clarify these ideas, let us consider the evaluation of the electron.Green's function in the atomic limit. We first consider only the configurations for a shell of equivalent $d$ (or $f$ ) electrons. As indicated in Sec. II, the atomic eigenope rators satisfy

$$
H b_{n}^{\dagger}(\alpha)=E_{n}(\alpha) b_{n}^{\dagger}(\alpha)
$$

By utilizing the properties of the Lie group ${ }^{46}$ associated with the electron states in an atomic shell, an orthonormal set of interconfiguration excitation operators can be constructed by forming

$$
\tilde{\phi}_{n}(\alpha, \beta)=b_{n-1}^{\dagger}(\alpha) I_{\ominus} b_{n}(\beta)
$$

where the operator $I_{\ominus}$ is the invariant of the filled shell. ${ }^{47}$ For a $d$ shell this may be written as

$$
I_{\ominus}=b_{10}\left({ }^{1} S\right) b_{10}^{\dagger}\left({ }^{1} S\right) \text {, }
$$

where ${ }^{1} S$ denotes the filled shell; also, all the $b_{n}$ are written with respect to some canonical ordering of the single-particle orbitals $c_{d s}$. Judd ${ }^{48}$ has discussed the utilization of this sort of invariant $I_{\ominus}$ in second-quantized treatments of $l^{n}$ atomic shells. The ICEE vectors $\tilde{\phi}_{n}(\alpha, \beta)$ can be normalized by using the thermal inner product

$$
\left\|\tilde{\phi}_{n}(\alpha ; \beta)\right\|^{2}=\left\langle\left\{\tilde{\phi}_{n}(\alpha, \beta), \tilde{\phi}_{n}^{\dagger}(\alpha, \beta)\right\}\right\rangle \text {. }
$$

We write the normalized ICEE vectors without the tilde, $\phi_{n}(\alpha, \beta)$. These vectors are precisely the orthonormal eigenvectors of $L$ (in the atomic limit), and they connect impurity configurations with a single-electron difference: using (2.2),

$$
L \phi_{n}(\alpha, \beta)=\epsilon_{n}(\alpha, \beta) \phi_{n}(\alpha, \beta) .
$$

This orthonormal set of eigenvectors of $L$ is complete in this case so we can expand the $c_{d s}$ ' $s$ in the atomic limit impurity Green's function using 
the standard eigenfunction expansion. The result for this case is

$$
\begin{aligned}
& \left(\left(c_{d s},(\omega-L)^{-1} c_{d s}\right)\right) \\
& =\sum_{\alpha \beta \eta} \frac{\left|\left(\left(c_{d s}, \phi_{n}(\alpha, \beta)\right)\right)\right|^{2}}{\omega-\epsilon_{n}(\alpha, \beta)} .
\end{aligned}
$$

For the atomic limit (3.14) is nothing more than a rewrite of (3.2), since it is possible in this case to compute directly the grand canonical partition function as implied in (3.2). The real utility of (3.14) is that the inner product can be written out in terms of equal time expectation values of related matrix elements of the inverse of (3.8) without the partition function. The evaluation of these equal time expectation values: by the fluctuation dissipation theorem ${ }^{49}$ yields self-consistency equations which reproduce the partition function and all the thermal averages. In the large interacting system, where no other tractable means exists for calculating the thermal averages, this formalism is potentially quite useful.

To ground these ideas in specifics, let us now specialize to the Anderson model. We first discuss the atomic limit $\left(V_{\overrightarrow{\mathrm{k}} d}=0\right)$. Because of the simplicity of this model we can enumerate the quantum states of $H_{0}-\mu N$. The impurity states are four in total: a no-electron state with energy zero, two oneelectron states with energy $\epsilon_{d s}$, and a single twoelectron state with energy $\epsilon_{d \uparrow}+\epsilon_{d \downarrow}+U$. The spectrum of the one-electron Green's function must reflect energy differences between those manyelectron states which differ by one electron. The only such differences are four in number: $\epsilon_{d \uparrow}$, $\epsilon_{d \downarrow}, \epsilon_{d \uparrow}+U$, and $\epsilon_{d \downarrow}+U$. For a given spin value $s$, the one-electron Green's function will always exhibit two poles whenever $U \neq 0$. This exact property is to be contrasted with the characteristics of the Hartree-Fock treatment which attempts to replace these two resonances for each spin direction with a single pole at $\epsilon_{d s}+U\left\langle n_{d \bar{s}}\right\rangle$, where $\left\langle n_{d \bar{s}}\right\rangle$ is the average occupation number. These two resonances at $\epsilon_{d s}$ and $\epsilon_{d s}+U$ are infinitely sharp in the atomic limit $\left(V_{\overrightarrow{\mathrm{k}} d}=0\right)$; we should expect their broadened form to be present in the interacting case for small $V_{\overrightarrow{\mathrm{k}} d}$.

Let us write out the operators (3.10) and (3.11) for this simple model. First,

$$
I_{\Theta}=c_{d \downarrow} c_{d \uparrow} c_{d \uparrow}^{\dagger} c_{d \downarrow}^{\dagger} .
$$

The operator acting between the one- and twoelectron configurations is

$$
\tilde{\phi}_{d s}^{(+)}=\phi_{2}\left({ }^{2} S ;{ }^{1} S\right)=c_{d \bar{s}}^{\dagger}\left(c_{d \bar{s}} c_{d s} c_{d s}^{\dagger} c_{d \bar{s}}^{\dagger}\right) c_{d \bar{s}} c_{i s},
$$

which may be simplified as $n_{d \bar{s}} c_{d s}$. A simple calculation shows that the normalized version of this operator is

$$
\phi_{d s}^{(t)}=\phi_{2}\left({ }^{2} S:{ }^{1} S\right)=n_{d \bar{s}} c_{d s} /\left(\left\langle n_{d \bar{s}}\right\rangle\right)^{-1 / 2} .
$$

It can be explicity verified that

$$
L \phi_{d s}^{(+)}=\left(\epsilon_{d s}+U\right) \phi_{d s}^{(+)} \text {. }
$$

The operator $c_{d s}$ changes the number of electrons from one to zero and is the only such operator, but it is not orthogonal to $\phi_{d s}^{(+)}$,

$$
\left(\left(c_{d s}, \phi_{d s}^{(+)}\right)\right)=\left\langle n_{d \bar{s}}\right\rangle^{1 / 2} .
$$

The Schmidt orthogonalization procedure will generate the required vector from a linear combination of $c_{d s}$ and $\phi_{d s}^{(+)}$. The normalized form of this vector is

$$
\phi_{d s}^{(-)}=\left(1-n_{d \bar{s}}\right) c_{d s} /\left(1-\left\langle n_{d \bar{s}}\right\rangle\right)^{1 / 2},
$$

which may be verified to have eigenvalue $\epsilon_{d s}$. The $( \pm)$ notation is less cumbersome for this model than (3.10) and is the same as Schrieffer' ${ }^{50}$ where the vectors $\phi_{d s}^{( \pm)}$have eigenvalues $E_{s}^{( \pm)}=\epsilon_{d s}+U / 2$ $\pm U / 2$. To simplify further the notation in the following we define the operator $N_{d s}^{(r)}$ to equal $n_{d s}$ when $r=+1$ and to give $1-n_{d s}$ when $r=-1$.

The reader familiar with Hubbard's analysis of his atomic Hamiltonian in the narrow band-metal context will recall that the operators $N_{d s}^{\left(\frac{r}{s}\right)} c_{d s}$ arose naturally in his study. The use of the inner product (3.6) and Judd's Lie group analysis suggest more profound properties of these two operators than may have been apparent in Hubbard's earlier work. The operators $\phi_{d s}^{(r)}$ are an orthonormal basis of eigenfunctions of $L$ containing an odd number of Fermion operators. The collection of such operators is dense in a Hilbert space ${ }^{51}$ and the one-electron operator $c_{d s}$ can be expanded in this orthonormal basis as

$$
c_{d s}=\sum_{r}\left\langle N_{d \bar{s}}^{(r)}\right\rangle^{1 / 2} \phi_{d s}^{(r)},
$$

giving the one-electron Green's function

$$
G_{d s}(\omega)=\sum_{r} \frac{\left\langle N_{d s}^{(r)}\right\rangle}{\omega-E_{s}^{(r)}} .
$$

The thermodynamic self-consistency conditions whose solutions generate the underlying grand canonical ensemble partition function are obtained from (3.20) as the two equations $(s= \pm 1)$

$$
\left\langle n_{d s}\right\rangle=\left(1-\left\langle n_{d \bar{s}}\right\rangle\right) f_{s}^{(-)}+\left\langle n_{d \bar{s}}\right\rangle f_{s}^{(+)},
$$

where $f_{s}^{(r)}=\left\langle\cdot \phi_{d s}^{\dagger(r)} \phi_{d s}^{(r)}\right\rangle$. The $f_{s}^{(r)}$ are calculcated using the standard Green's function techniques. For the atomic limit, the $f_{s}^{(r)}$ are simply Fermi functions

$$
f_{s}^{(r)}=\left(e^{\beta E_{s}^{(r)}}+1\right)^{-1}
$$

and the solution of the linear system of equations is immediate. It is then a simple matter to de- 
termine all of the thermal properties of the impurity from the solutions for $\left\langle n_{d \uparrow}\right\rangle$ and $\left\langle n_{d \downarrow}\right\rangle$.

The zero-field susceptibility $\chi_{T}$ is obtained by calculating the magnetic moment to linear order in the field, in terms of the $f_{s}^{(+)}$functions, and it is given by

$$
\begin{aligned}
\chi_{T}=\frac{-2 \mu_{B}^{2}}{1-f^{(-)}+f^{(+)}} & {\left[\left(\frac{\partial f^{(-)}}{\partial E^{(-)}}\right) \frac{1-f^{(+)}}{\left(1+f^{(-)}-f^{(+)}\right)}\right.} \\
& \left.+\left(\frac{\partial f^{(+)}}{\partial E^{(+)}}\right) \frac{f^{(-)}}{\left(1+f^{(-)}-f^{(+)}\right)}\right],
\end{aligned}
$$

where we assume $g=2$ and $\mu_{B}$ is the Bohr magneton.

If $E_{s}^{(-)}<0$ and $E_{s}^{(+)}>0$, which corresponds to the one-electron states being the ground state of the isolated atom, the impurity is magnetic and shows a Curie susceptibility

$$
\chi_{T} \approx \mu_{B}^{2} / k_{B} T
$$

for any temperature $k_{B} T$ low compared to $\left|E_{s}^{(+)}\right|$ or $\left|E_{s}^{(-)}\right|$. If $E_{s}^{(+)}$is negative or if $E_{s}^{(-)}$is positive, the susceptibility goes to zero as $T \rightarrow 0$, in the first case because the ground state is the twoelectron singlet state, and in the second case, because the no-electron state is lowest in energy $(U \rightarrow 0)$ and the impurity is unoccupied at low temperatures. One of these cases is apparently realized for dilute ThCe alloys..$^{52}$

In terms of atomic values of these parameters $\epsilon_{s}$ and $U$, atomic hydrogen values yield the magnetic type of impurity $\epsilon_{s}<\epsilon_{\uparrow}+\epsilon_{\downarrow}+U$, while atomic helium values for $\epsilon$ and $U$ yield the nonmagnetic singlet ground-state atom.

For any of these cases, the heat capacity at constant volume for the impurity in equilibrium with a particle bath at chemical potential $\mu$,

$$
C_{V}=\frac{\partial}{\partial T}\langle H-\mu N\rangle_{V},
$$

will exhibit a Schottky anomaly with a heat-capacity peak occurring at a temperature $T_{p}$ which is defined as

$$
k_{B} T_{p} \approx \frac{1}{2} \min \left\{\left|\epsilon_{d s}\right|,\left|\epsilon_{d s}+U\right|\right\} .
$$

For this simple isolated-atom model, if the impurity is magnetic the entropy change from low to high temperatures will be $k_{B} \ln 2$, while for the nonmagnetic impurity the change is $k_{B} \ln 4$. In the presence of an interaction between impurity and host the scattering of the conduction electrons is related to the $t$ matrix which can be written

$$
t_{\overrightarrow{\mathrm{k}}, \overrightarrow{\mathrm{k}}{ }^{\prime}}(\omega)=\sum_{s} V_{\overrightarrow{\mathrm{k}} d} V_{\overrightarrow{\mathrm{k}}^{\prime} d} G_{d s}(\omega) .
$$

An approximation for this can be obtained in what might be termed the atomic limit if we put the atomic limit $G_{d s}(\omega)$ into the expression for the $t$ matrix. For all $U \neq 0$, the $t$ matrix will have resonances at $\omega=E_{s}^{( \pm)}$and will never have a single resonance.

Let us now examine corrections to this atomic limit $t$ matrix in an heuristic fashion with a simple application of perturbation theory. If there is no lifetime broadening of the impurity ICEE's, then the scattering $t$ matrix has an imaginary part,

$$
\begin{aligned}
\operatorname{Im} t(\omega)=\sum_{s} V_{\overrightarrow{\mathbf{k}} d} V_{\overrightarrow{\mathbf{k}}^{\prime} d}[(1 & \left.-\left\langle n_{d \bar{s}}\right\rangle\right) 2 \pi \delta\left(\omega-\epsilon_{d s}\right) \\
& \left.+\left\langle n_{d \bar{s}}\right\rangle 2 \pi \delta\left(\omega-\epsilon_{d s}-U\right)\right],
\end{aligned}
$$

consisting of two $\delta$ functions. In this approximation, unless one of the ICEE's is at the Fermi energy there would be no resistivity from this impurity at low temperatures.

We next examine the effect of coupling the impurity states to the conduction electrons in two steps: first, we consider the effect of lifetime broadening,

$$
\Delta=\pi N(0) V_{\overrightarrow{\mathrm{k}} d}^{2},
$$

and then we examine in second-order perturbation theory the change of the term energies of each configuration of the impurity. If the impurity ICEE's are lifetime broadened, the normalized resistivity

$$
R / R_{0}=\pi N(0) \operatorname{Im} t(0)
$$

is given by

$$
\frac{R}{R_{0}}=\sum_{s}\left[\frac{\left(1-\left\langle n_{d \bar{s}}\right\rangle\right) \Delta^{2}}{\epsilon_{d s}^{2}+\Delta^{2}}+\frac{\left\langle n_{d \bar{s}}\right\rangle \Delta^{2}}{\left(\epsilon_{d s}+U\right)^{2}+\Delta^{2}}\right] .
$$

In the usual choice of impurity parameters, $U>-\epsilon_{d s},\left|E_{s}^{(r)}\right|>\Delta$, and the $\Delta^{2}$ in the denominators can be neglected, and we can write the lowestorder resistivity in terms of an effective exchange parameter using (1.6):

$$
\frac{R}{R_{0}}=\left[\pi N(0) J_{\text {eff }}\right]^{2}\left(1+\frac{2 \epsilon_{d s}\left(\epsilon_{d s}+U\right)}{U^{2}}\right),
$$

where we have assumed $\kappa_{B} T<\left|\epsilon_{d s}^{(r)}\right|$ and thus $\left\langle n_{d s}\right\rangle$ $=\frac{1}{2}$. If one of the ICEE energies is small, say $0<\epsilon_{d s}+U \ll-\epsilon_{d s}$, then the resistivity is dominated by the $(+)$ resonance which is closest to the Fermi energy. In this case the resistivity becomes

$$
\frac{R}{R_{0}} \approx \frac{\left\langle n_{d \bar{s}}\right\rangle \Delta^{2}}{\left(E_{s}^{(+)}\right)^{2}+\Delta^{2}}
$$

If $E_{s}^{(+)}$is very small the resistivity would be close to the unitarity limit for this channel, while if $\left|E_{s}^{(t)}\right|$ is comparable to or larger than $\Delta$ the resistivity could be quite small.

One of the major results of this work, which we 
derive below in second-order perturbation theory and verify in our many-body approximation scheme later, is the fact that the coupling of the impurity configurations to the conduction electrons gives rise to a temperature dependent ICEE shift $\delta E_{s}^{(r)}$. In the case we are studying the ICEE becomes

$$
e_{1 s}^{(+)}=\epsilon_{d s}+U-\delta E_{s}^{(+)} \text {. }
$$

It will be shown later that, if $\epsilon_{d s}+U>0$ and small and $\epsilon_{d s}<0$ and large, then $\delta E_{s}^{(+)}$is positive and is logarithmically increasing at $T \rightarrow 0$. In Sec. IV, it is demonstrated that this circumstance can recover a Kondo-like resistivity with a quite different effective coupling constant than is usually derived.

As will be shown later,

$$
\delta E_{s}^{(+)}=\frac{\Delta}{\pi} \ln \left(\frac{1.13\left|\epsilon_{d s}\right|}{k_{B} T}\right)
$$

in a certain temperature range where $\delta E_{s}^{(+)}$is small. Expanding the resistivity to lowest order in the shift yields

$$
\begin{aligned}
\frac{R}{R_{0}}=\sum_{s} \frac{\left\langle n_{d \bar{s}}\right\rangle \Delta^{2}}{\left(\epsilon_{d s}+U\right)^{2}+\Delta^{2}}[1 & +N(0) J_{\text {eff }}^{\prime} \\
& \left.\times \ln \left(\frac{1.13\left|\epsilon_{d s}\right|}{k_{B} T}\right)+\cdots\right]
\end{aligned}
$$

where

$$
N(0) J_{\mathrm{eff}}^{\prime}=\frac{2}{\pi} \frac{\left(\epsilon_{d s}+U\right) \Delta}{\left(\epsilon_{d s}+U\right)^{2}+\Delta^{2}} .
$$

This is a Kondo-like result, but with a very different and possibly quite small $J_{\text {eff }}^{\prime}$.

An experimental verification of the functional form in (3.36) and (3.37) is provided by pressure studies $^{53}$ of the dependence of the derivative of the resistivity with respect to $\ln T$. Conventional Kondo explanations ${ }^{54}$ of this effect require extremely complex variations of $T_{k}$ with pressure. This work results in a very simple prediction which is well reproduced by experiment. If we assume that pressure changes the ICEE in a fashion that varies linearly with pressure, then the theory predicts that

$$
\frac{\partial \rho}{\partial \ln T} \propto \frac{\epsilon_{d}+U}{\left[\left(\epsilon_{d}+U\right)^{2}+\Delta^{2}\right]^{2}}+\text { const. }
$$

Using the assumed linear pressure variation of the ICEE we may write this as

$$
\frac{\partial \rho}{\partial \ln T}=\frac{A X}{\left(1+X^{2}\right)^{2}}+\text { const }
$$

where

$$
X=X_{0}-P / P_{0}
$$

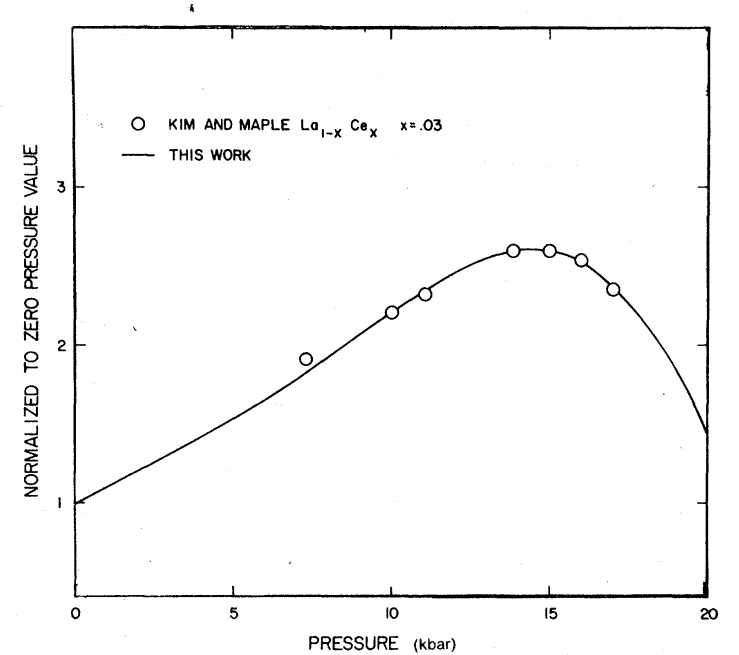

FIG. 1. This figure shows the pressure dependence of the resistivity derivative $\partial \rho / \partial \ln T$. The derivative is normalized to 1 at zero pressure. The open circles are experimental points and the solid line is the simple theory. See text for full explanation.

Figure 1 of this paper shows a fit of this result to experimental data ${ }^{55}$ for $A=8.16, X_{0}=1.6$, and $P_{0}=14.66$, and negligible additive constant. Such a close fit from such a simple assumption concerning the pressure change of the ICEE at the very least lends credibility to the considerations of this work.

In Sec. IV and the appendices we will discuss the temperature range in which this result obtains, but first it will be demonstrated that such a shift arises naturally in second-order perturbation theory of the impurity configuration terms.

We seek to apply the perturbation $V(1.3)$ to states of $H_{0}(1.2)$. In particular, we seek to determine the changes in the energies of

$$
\begin{aligned}
& \left|\psi_{0}\right\rangle=\left|\psi_{F}\right\rangle, \\
& \left|\psi_{1 s}\right\rangle=c_{d s}^{\dagger}\left|\psi_{F}\right\rangle, \quad s= \pm 1, \\
& \left|\psi_{2}\right\rangle=c_{d \downarrow}^{\dagger} c_{d \downarrow}^{\dagger}\left|\psi_{F}\right\rangle,
\end{aligned}
$$

where $\left|\psi_{F}\right\rangle$ is any conduction-electron state of the host. One almost immediately observes that first-order perturbation theory produces no change, and we must evaluate the second-order contributions. For the impurity vacuum state $\left|\psi_{F}\right\rangle$ there is only one intermediate state $c_{d s}^{\dagger} c_{\mathrm{ks}}\left|\psi_{F}\right\rangle$ and the change in the energy of impurity vacuum is

$$
\Delta E_{2}\left(\psi_{0}\right)=-\sum_{\overrightarrow{\mathrm{k}}} \frac{V_{\overrightarrow{\mathrm{k}} d}^{2} f_{\overrightarrow{\mathrm{k}} s}}{\epsilon_{d s}-\epsilon_{\overrightarrow{\mathrm{k}} s}} .
$$

In arriving at this result we have followed the standard method of doing the perturbation calculation for each definite many-electron state in 
which $f_{\overrightarrow{\mathrm{k}} s}=0$ or 1 and using $\underset{f_{\mathrm{k} s}}{2}=f_{\overrightarrow{\mathrm{k}} s}$. After obtaining the expression for any particular $\left|\psi_{F}\right\rangle$ we can then take the thermal average of both sides of the equation and get the temperature-dependent shift. Upon averaging the $f_{\overrightarrow{\mathrm{k} s}}$ becomes the conductionelectron Fermi function. For the $\left|\psi_{1 s}\right\rangle$, there are two types of intermediate states,

$$
c_{d \bar{s}}^{\dagger} c_{\mathrm{k} \bar{s}} c_{d s}^{\dagger}\left|\psi_{F}\right\rangle \text { and } \underset{c_{\mathrm{k}} \bar{s}}{\dagger} c_{d \bar{s}} c_{d s}^{\dagger}\left|\psi_{F}\right\rangle \text {. }
$$

These give a perturbed energy of

$$
\Delta E_{2}\left(\psi_{1 s}\right)=-\sum_{\overrightarrow{\mathrm{k}}} \frac{V_{\overrightarrow{\mathrm{k}} d}^{2} f_{\overrightarrow{\mathrm{k}} \bar{s}}}{\epsilon_{d \bar{s}}+U-\epsilon_{\overrightarrow{\mathrm{k}}} \bar{s}}+\sum_{\overrightarrow{\mathrm{k}}} \frac{V_{\overrightarrow{\mathrm{k}} d}^{2}\left(1-f_{\overrightarrow{\mathrm{k}} s}\right)}{\epsilon_{d s}-\epsilon_{\overrightarrow{\mathrm{k}} s}} .
$$

For the two-particle state, the intermediate state is $c_{\overrightarrow{\mathrm{k} s}}^{\dagger} c_{d s}^{\dagger}\left|\psi_{F}\right\rangle$ and the energy shift is

$$
\Delta E_{2}\left(\psi_{2}\right)=+\sum_{\overrightarrow{\mathrm{k}} s} \frac{V_{\overrightarrow{\mathrm{k}} d}^{2}\left(1-f_{\overrightarrow{\mathrm{k}} s}\right)}{\epsilon_{d s}+U-\epsilon_{\overrightarrow{\mathrm{k}} s}} .
$$

The result for the ICEE is

$$
e_{1 s}^{(r)}=E_{s}^{(r)}-\left(\sum_{\overrightarrow{\mathrm{k}}} \frac{V_{\overrightarrow{\mathrm{k}} d}^{2} f_{\overrightarrow{\mathrm{k}} \bar{s}}}{\epsilon_{d \bar{s}}+U-\epsilon_{\overrightarrow{\mathrm{k}}} \bar{s}}-\sum_{\overrightarrow{\mathrm{k}}} \frac{V_{\overrightarrow{\mathrm{k}} d}^{2} f_{\overrightarrow{\mathrm{k}} \bar{s}}}{\epsilon_{d \bar{s}}-\epsilon_{\overrightarrow{\mathrm{k}}} \bar{s}}\right),
$$

plus terms like the $f_{\overrightarrow{\mathrm{k}} s}$ terms but with $f_{\overrightarrow{\mathrm{k}} s}$ replaced by 1 . These latter terms we neglect as being incorporated into new values of $e_{1 s^{\circ}}^{(r)}$ In Sec. IV, we shall recover this same result in our degenerate Feenberg perturbation theory and there will write the shift as a function $\Lambda^{\prime}$, where

$$
\begin{aligned}
\frac{\Delta}{\pi} \Lambda^{\prime} \approx & \operatorname{Re} \sum_{\overrightarrow{\mathrm{k}}} \frac{V_{\mathrm{k} d}^{2} f_{\overrightarrow{\mathrm{k}} \bar{s}}}{\epsilon_{d \bar{s}}+U-i \Delta-\epsilon_{\overrightarrow{\mathrm{k}} \bar{s}}} \\
& -\operatorname{Re} \sum_{\overrightarrow{\mathrm{k}}} \frac{V_{\overrightarrow{\mathrm{k}} d}^{2} f_{\overrightarrow{\mathrm{k}} \bar{s}}}{\epsilon_{d \bar{s}-\epsilon_{\overrightarrow{\mathrm{k}}-\bar{s}}-i \Delta}}
\end{aligned}
$$

and in anticipation of results in Sec. V I have included lifetime broadening in the intermediate states. As will be discussed more fully in that section, $\Lambda^{\prime}$ displays a $\log (T)$ behavior for high temperature and a $1-T^{2}$ behavior at low temperatures.

Before closing this section, let us make some brief comments about the impurity susceptibility in the lifetime broadened atomic limit. Inserting a constant lifetime broadening into each ICEE resonance in the impurity Green's function changes the

$$
f_{s}^{(r)}=\left\langle\phi_{d s}^{\dagger(r)} \phi_{d s}^{(r)}\right\rangle
$$

from the usual Fermi functions into something characteristic of a system coupled to a system with a constant density of states near the Fermi energy, i.e.,

$$
f_{s}^{(r)}=\frac{1}{2}-\frac{1}{\pi} \operatorname{Im} \psi\left[\frac{1}{2}+\frac{\beta}{2 \pi}\left(\Gamma+i E_{s}^{(r)}\right)\right],
$$

where $\psi$ is the digamma function. If $\Gamma$ is much larger than $\left|E_{s}^{(r)}\right|$, a straightforward evaluation of (3.24) using (3.47) gives exactly the strong coupling result (1.10). At this point it is worthwhile observing that in Appendix D we use the thermodynamic Hilbert space formalism introduced elsewhere to illustrate why HFA poorly indicates the presence of an ICEE near the Fermi energy.

\section{INTERACTING IMPURITY AND CONDUCTION ELECTRONS}

In order to evaluate the impurity Green's function when interactions are present, it becomes necessary to deal with nondiagonal secular matrices of $L$. The most useful technique for studying this case has been the Feenberg point of view. To illustrate the approximations to be used below and review ${ }^{56}$ the essential aspects of the method let us adopt

$$
H_{n n^{\prime}}=\delta_{n n^{\prime}} \epsilon_{n}+V_{n n^{\prime}}
$$

as notation for the matrix elements of a Hamiltonian $H$ or $L$ operator and examine formulas for diagonal and off-diagonal matrix elements of the resolvent, $(\omega-H)_{n, n^{\prime}}^{-1}$. For conceptual definiteness in this application the indices $n, n^{\prime}$ (and below $l, l^{\prime}$, etc.) may be thought of as labeling quantum numbers of many-electron correlated states as well as one-electron states. The essential point of Feenberg's method of perturbation theory has become the modus operandi of band-structure calculations. This is the property of truncating the secular equation in some fashion and solving the remaining determinant exactly. This means that the self-energy expressions in the Feenberg picture always include the summation restrictions which arise in any determinant expression. In the discussion that follows, our approximations will rely on two special properties of this problem. First we study a weak coupling limit for the $V$ 's, and thus include only $V_{\vec{k}}^{2}$ terms in the self-energy. Actually, this is not precisely the manner in which to describe the calculation scheme. Because of the infinite number of conduction-electron states our secular equation must be infinite dimensional. This causes no real difficulty because the conduction-electron part of the secular equation is block diagonal with few simple matrix elements to the impurity operators. The offdiagonal matrix elements connecting vectors containing conduction electrons and those few vectors which are wholly impurity states are found only in a few rows or columns. As we begin with the diagonal secular equation for the atomic sub- 
spaces, the resulting matrix is essentially diagonal blocks connected by a few rows and columns, all other entries being zero. This situation might be described ${ }^{57}$ as a generalized bordered determinant. When the matrix elements in these borders are already small, the matrix elements of the resolvent can be approximated by

$$
(\omega-H)_{n n}^{-1}=\left[\omega-\epsilon_{n}-\Sigma(n, w)\right]^{-1},
$$

where the self-energy $\Sigma(n, \omega)$ is written

$$
\Sigma(n, \omega)=\sum_{l(\neq n)} \frac{\left|V_{n l}\right|^{2}}{\omega-e_{l}(n, \omega)}+\cdots,
$$

and the reduced self-energy is

$$
e_{l}(n, \omega)=\epsilon_{l}+\sum_{l_{1}(\neq n l)} \frac{\left|V_{l i_{1}}\right|^{2}}{\omega-e_{l_{1}}(n l, \omega)}+\cdots .
$$

The further reduced self energies $e_{l_{r}}\left(n l_{1} \ldots l_{r-1}, \omega\right)$ have similar expressions, but more restrictions. As we seek a quasiparticle approximation for each resonance of the final Green's function, the form of the higher-order reduced self-energies will not be important. If the summation restrictions involve the conduction electrons, they can be ignored, ${ }^{58}$ but the atomic summation restrictions must be obeyed. Within this approximation scheme the off-diagonal matrix elements of the resolvent will be written as

$$
(\omega-H)_{l l^{\prime}}^{-1}=\left[\omega-e_{l}(\omega)\right]^{-1} V_{l l^{\prime}}\left[\omega-e_{l^{\prime}}(l, \omega)\right]^{-1} .
$$

To determine the self-energies and the intermediate states which must go into the Feenberg expressions for the self-energy, it is necessary to construct the secular equation for $L$ with respect to an orthonormal basis. We adopt as the initial vectors in this basis the conduction-electron operators $c_{\overrightarrow{k s}}$ and the impurity eigenvectors $\phi_{d s}^{(r)}$. We derive the various rows of the secular equation for the Anderson model $L$ from the standard equations of motion. The first set of rows is given by

$$
L c_{\overrightarrow{\mathrm{k} s}}=\epsilon_{\overrightarrow{\mathrm{k} s}} c_{\overrightarrow{\mathrm{k} s}}+\sum_{r= \pm 1} V_{\overrightarrow{\mathrm{k}} d}\left\langle N_{d \vec{s}}^{(r)}\right\rangle^{1 / 2} \phi_{d s}^{(r)} .
$$

As the vectors in this equation are orthonormal their coefficients represent the matrix elements of $L$ which will be used in constructing the selfenergy expressions.

Note, in particular, that the matrix element which will contribute to the lifetime broadening of the resonance associated with $\phi_{d s}^{(r)}$ is modulated by a population factor. While in the $S$-shell model being studied here these population factors produce no significant lifetime narrowing of the resonances, similar factors arising in a many elec- tron model using the $d$ or $f$ shell $\phi_{n}(\alpha, \beta)$ could give rise to significant lifetime narrowing for half-filled shells.

Since $L$ is a symmetric operator we must expect the $\phi_{d s}^{(r)}$ rows of the secular equations to have symmetric matrix elements with each $c_{\vec{k} s}$. Operating with $L$ on $\phi_{d s}^{(r)}$ yields

$$
\begin{aligned}
L \phi_{d s}^{(r)}= & E_{s}^{(r)} \phi_{d s}^{(r)}+\sum_{\overrightarrow{\mathrm{k}}} V_{\overrightarrow{\mathrm{k}} d} N_{d \bar{s}}^{(r)} c_{\overrightarrow{\mathrm{k} s}} /\left\langle N_{d \bar{s}}^{(r)}\right\rangle^{1 / 2} \\
& +\frac{r}{\left\langle N_{d \bar{s}}^{(r)}\right\rangle^{1 / 2}} \sum_{\overrightarrow{\mathrm{k}}} V_{\overrightarrow{\mathrm{k}} d}\left(c_{d \vec{s}}^{\dagger} c_{\overrightarrow{\mathrm{k}} \bar{s}}-c_{\overrightarrow{\mathrm{k}} \bar{s}}^{\dagger} c_{d \bar{s}}\right) c_{d s} .
\end{aligned}
$$

This expression does not manifest the desired symmetry and, in fact, does not directly involve $c_{\overrightarrow{k s}}$ alone as a vector on the right-hand side. Application of the Schmidt orthogonalization procedure to the second term yields the desired results:

$$
\begin{aligned}
L \phi_{d s}^{(r)}= & E_{s}^{(r)} \phi_{d s}^{(r)}+\left\langle N_{d \bar{s}}^{(r)}\right\rangle^{1 / 2} \sum_{\overrightarrow{\mathrm{k}}} V_{\overrightarrow{\mathrm{k}} d} c_{\overrightarrow{\mathrm{k}} s} \\
& +r\left\langle N_{d \bar{s}}^{(-r)}\right\rangle^{1 / 2} \sum_{\overrightarrow{\mathrm{k}}} V_{\overrightarrow{\mathrm{k}} d} \phi_{d \overrightarrow{\mathrm{k} s}} \\
& +\frac{r}{\left\langle N_{d \bar{s}}^{(r)}\right\rangle^{1 / 2}} \sum_{\overrightarrow{\mathrm{k}}} V_{\overrightarrow{\mathrm{k}} d} c_{d \bar{s}}^{\dagger} c_{\overrightarrow{\mathrm{k}} \bar{s}} c_{d s} \\
& -\frac{r}{\left\langle N_{d \bar{s}}^{(r)}\right\rangle^{1 / 2}} \sum_{\overrightarrow{\mathrm{k}}} V_{\overrightarrow{\mathrm{k}} d} c_{\overrightarrow{\mathrm{k}} \bar{s}}^{\ddagger} c_{d \bar{s}} c_{d s},
\end{aligned}
$$

where the new vector $\phi_{d \overrightarrow{k s}}$ is defined as

$$
\left.\phi_{d \overrightarrow{\mathrm{k} s}}=\left(n_{d \bar{s}}-\left\langle n_{d \bar{s}}\right\rangle\right) c_{\overrightarrow{\mathrm{k} s}} /\left\langle n_{d \bar{s}}\right\rangle\left(1-\left\langle n_{d \bar{s}}\right\rangle\right)\right]^{1 / 2} .
$$

Now the first three terms of this equation form an orthonormal basis set, but the last two terms are not orthogonal to the vector $\phi_{d s}^{(r)}$. This means that we must first determine the overlap between $\phi$ 's and the vectors $c_{d \bar{s}}^{\dagger} c_{\vec{k} \bar{s}} c_{d s}$, and $c_{\mathrm{k}}^{\dagger} c_{d \bar{s}} c_{d s}$, and then construct appropriate linear combinations of the vectors to yield an orthonormal basis. The essential outlines of this procedure are given in Appendix A. The effect on the above equation is to add to the diagonal matrix element $E_{s}^{(r)}$ a temperature-dependent energy shift $\Delta E_{s}^{(r)}$ which arises from the orthogonalization of the different threeoperator vectors.

This temperature-dependent energy shift of the ICEE can be thought of as arising from the coupling of the conduction electrons to the impurity. If there were no coupling of the impurity to the host in this energy range, the energy of a transition from $\left|d^{n-1} ; \alpha\right\rangle$ to $\left|d^{n} ; \beta\right\rangle$ would only be $\epsilon_{n}\left(\alpha^{\prime}, \beta\right)$. However, with the conduction electrons mixed into the impurity states the transition is not possible without corresponding transitions of conductionelectron states. The temperature dependence of the shift merely reflects the thermal distribution of the intermediate conduction-electron states which are mixed into the impurity configurations. 
If lifetime broadening of the intermediate states is neglected the shift at $T=0$ is just the formal energy shift from second-order perturbation theory (3.41).

In Appendix A it is shown that the energy shift can be written as

$$
\Delta E_{s}^{(r)}=-\frac{\Delta}{\pi} \frac{\left\langle n_{d s}\right\rangle}{\left\langle N_{d \bar{s}}^{(r)}\right\rangle}\left(\lambda_{s}^{(+)}-\frac{\left(1-\left\langle n_{d s}\right\rangle\right)}{\left\langle n_{d s}\right\rangle} \lambda_{s}^{(-)}\right),
$$

where the parameters $\lambda_{s}^{(r)}$ are positive definite quantities defined by

$$
-\frac{\Delta}{\pi}\left\langle N_{d s}^{(r)}\right\rangle^{1 / 2} \lambda_{s}^{(r)}=\sum_{\overrightarrow{\mathrm{k}}} V_{\overrightarrow{\mathrm{k}} d}\left\langle c_{\overrightarrow{\mathrm{k}}}^{\dagger} \bar{s} \phi_{d \bar{s}}^{(r)}\right\rangle
$$

This latter definition is related to the equal time expectation value of an off-diagonal matrix element of the resolvent,

$$
G_{\mathrm{k} d \bar{s}}^{(r)}(\omega)=\left(\left(c_{\overrightarrow{\mathrm{k}}} \bar{s},(\omega-L)^{-1} \phi_{d \bar{s}}^{(r)}\right)\right) .
$$

Since this off-diagonal matrix element of the resolvent will depend on the same self-energy as the $G_{d s}(\omega)$, the temperature dependence of the shift must be determined self-consistently. This is done in Appendix B. If we define as $e_{s}^{(r)}(\omega)$ the energy part of the denominator of the impurity Green's function,

$$
\left(\left(\phi_{d s}^{(r)},(\omega-L)^{-1} \phi_{d s}^{(r)}\right)\right)=\left[\omega-e_{s}^{(r)}(\omega)\right]^{-1},
$$

then the off-diagonal Green's function can be written in the weak coupling limit as

$$
G_{\overrightarrow{\mathrm{k}} d \vec{s}}^{(r)}=\frac{\left\langle N_{d s}^{(r)}\right\rangle^{1 / 2} V_{\overrightarrow{\mathrm{k}} d}}{\left(\omega-\epsilon_{\mathrm{k} s}\right)\left[\omega-e_{s}^{(r)}(\omega)\right]} .
$$

In terms of the resonance self-energy $\Sigma_{d s}^{(r)}(\omega)$ and the shift of the energy, $e_{s}^{(r)}$ is defined as

$$
e_{s}^{(r)}(\omega)=E_{s}^{(r)}+\Delta E_{s}^{(r)}+\Sigma_{d s}^{(r)}(\omega) .
$$

If one were to approximate the impurity resonance self-energy $\Sigma_{d s}^{(r)}$ by neglecting its real part and giving it a constant imaginary part, then the impurity response would be characterized by the presence of two broadened resonances at $E_{s}^{(r)}+\Delta E_{s}^{(r)}$ whose energies change with temperature. Such a simple approximation can already semiquantitatively account for some of the features of the dilute magnetic alloy problem. As will be discussed below and in more detail in Appendix B, the temperature dependence of the $\lambda_{s}^{(r)}$ is of the form $-\ln T$ at high temperatures and $1-T^{2}$ at low temperatures. For certain values of the physical parameters one of the resonances $e^{(r)}$ can approach the Fermi energy as $T \rightarrow 0$. This can give rise to the increase and saturation of the resistivity, the thermopower peaks, and Schottky-like anomalies in the heat capacity as well as other observed effects.
Actually there is no justification for neglecting the real part of the self-energy, even in the weakcoupling limit since it gives a contribution which is of the same size as the shift $\Delta E_{s}^{(r)}$. Accordingly we must study the real part of the self-energy. As detailed in Appendix A, the derivation of the impurity resonance energy $e_{s}^{(r)}$ yields in the weak coupling limit

$$
e_{s}^{(r)}=E_{s}^{(r)}-\frac{\Delta}{\pi} \frac{\left\langle n_{d s}\right\rangle}{\left\langle N_{d \bar{s}}^{(r)}\right\rangle} \Lambda_{s}+\gamma(\omega)+\frac{Y_{s}(\omega)}{\left\langle N_{d \bar{s}}^{(r)}\right\rangle},
$$

where $\gamma(\omega)$ is the usual one-electron self-energy familiar from previous studies of this model,

$$
\gamma(\omega)=\sum_{\overrightarrow{\mathrm{k}}} \frac{V_{\mathrm{k}}^{2}}{\omega-\epsilon_{\overrightarrow{\mathrm{k}} s}} \approx \pm i \Delta .
$$

The coefficient $\Lambda_{s}$ is

$$
\Lambda_{s}=\lambda_{s}^{(+)}-\frac{1-\left\langle n_{d s}\right\rangle}{\left\langle n_{d s}\right\rangle} \lambda_{s}^{(-)} .
$$

The remaining contribution $Y_{s}(\omega)$ is derived in Appendix $\mathrm{A}$ and is

$$
\begin{aligned}
Y_{s}(\omega)= & \left(1-\left\langle n_{d s}\right\rangle\right) f_{\bar{s}}^{(-)} \gamma(\omega)+\left\langle n_{d s}\right\rangle f_{\bar{s}}^{(+)} \gamma\left(\omega-2 \epsilon_{d}-U\right) \\
+ & \frac{\Delta}{\pi}\left[\left(\left\langle n_{d s}\right\rangle-\left\langle n_{d \bar{s}}\right\rangle \mathscr{g}_{D}\left(\omega, \Delta^{\prime}\right)\right.\right. \\
& \left.-\left(1-\left\langle n_{d s}\right\rangle-\left\langle n_{d \bar{s}}\right\rangle\right) g_{D}\left(\omega-2 \epsilon_{d}-U, \Delta^{\prime}\right)\right] .
\end{aligned}
$$

Here $f_{s}^{(r)}=\left\langle\phi_{d s}^{\dagger(r)} \phi_{d s}^{(r)}\right\rangle$ and the function $g_{D}(E, \Gamma)$ depends explicitly on the cutoff of the conductionband density of states,

$$
g_{D}(E, \Gamma)=\int_{-D}^{D} \frac{f_{0}(\epsilon) d \epsilon}{E-i \Gamma-\epsilon},
$$

and $f_{0}(\epsilon)$ is here the Fermi function. The properties of this function $\mathscr{g}_{D}$ are discussed in Appendix C. As noted there, at high temperatures $\mathscr{I}_{D}$ has a $\ln T$ dependence, but in all cases depends logarithmically on the cutoff i.e., $g_{D} \sim \ln D$ for large $D$. For purposes of continuity, let us note that if we write

$$
e_{s}^{(r)}=e_{1 s}^{(r)}+i e_{2 s}^{(r)},
$$

then the shift parameters $\lambda_{s}^{(r)}$ can also be expressed in terms of $g_{D}$ to order $1 / D$ in the weak $-V$ coupling limit, that is, as shown in Appendix B,

$$
\lambda_{s}^{(r)}=g_{D}\left(e_{1 s}^{(r)}, e_{2 s}^{(r)}\right)+O(1 / D) .
$$

We now consider what tractable approximations can be made on these expressions which will yield simple behavior. The essential idea that we use is to seek a Lorentzian approximation for each interconfigurational energy peak of the one-electron Green's function. Because the imaginary parts of the self-energy expressions are only weakly dependent on $\omega$ for energies near the cen- 
ter of the conduction band, their $\omega$ dependence is not too important. We simply write as constants

$$
e_{1 s}^{(r)}=E_{s}^{(r)}+\delta E_{s}^{(r)}, \quad e_{2 s}^{(r)}=\Gamma_{s}^{(r)},
$$

and set the frequencies in $\gamma_{s}(\omega)$ equal to $e_{1 s}^{(r)}$, and determine the expression for $\delta E_{s}^{(r)}$. After a certain amount of algebra very pleasant cancellations occur and we find that to lowest order in $\Delta$

$$
\delta E_{s}^{(r)}=-\frac{\Delta}{\pi}\left(\lambda_{s}^{(+)}-\lambda_{s}^{(-)}\right)+O\left(\frac{1}{D}\right) .
$$

This is an extremely satisfying result from a physical point of view because it is independent of the cutoff $D$ when $D$ is large, and depends on the impurity parameters only. Thus, the magnetic impurity problem is properly an impurity effect in that the temperature of the shift depends only on host density of states and impurity energies and not on the band width $D$. Writing

$$
\Lambda_{s}^{\prime}=\lambda_{s}^{(+)}-\lambda_{s}^{(-)},
$$

one finds that the temperature behavior of the impurity resonances is determined by two temperatures $T^{(r)}$ which are defined as

$$
k_{B} T^{(r)}=\frac{1}{\pi}\left[\left(E_{s}^{(r)}+\delta E_{s}^{(r)}\right)^{2}+\left(\Gamma_{s}^{(r)}\right)^{2}\right]^{1 / 2} .
$$

The weak temperature dependence of the $T^{(r)}$ due to the $\delta E_{s}^{(r)}$ is not important for the cases we consider. The analytic behavior of $\Lambda_{s}^{\prime}$ is quite simple in three ranges. First, consider $T^{(-)}>T^{(+)}$; this can correspond to $\epsilon_{d s} \ll 0$ and $\epsilon_{d s}+U \gtrsim 0$. This means in the $S$-shell model that the no-electron configuration is very high in energy, the two oneelectron configurations are lowest in energy, but that the two-electron configuration is close to the one-electron states in energy. If the temperature $T$ is greater than either $T^{(+)}$or $T^{(-)}$, Appendix A shows that $\Lambda_{s}^{\prime}$ is zero to order $1 / D$ and there is no shift of the ICEE with temperature. If the temperature is lower than $T^{(-)}$, but still higher than $T^{(+)}$, the $\lambda^{(-)}$is almost constant while the $\lambda^{(+)}$still varies logarithmically. For this intermediate temperature range, $T^{(+)}<T<T^{(-)}$, the ICEE shift will be logarithmic in $T$ :

$$
\Lambda^{\prime} \approx \ln \left(\frac{3.56 T^{(-)}}{T}\right)-\frac{\pi^{2}}{6}\left(\frac{T}{T^{(-)}}\right)^{2} .
$$

At low temperatures, that is, $T$ lower than both $T^{(+)}$and $T^{(-)}$, both $\lambda^{(r)}$ are almost constant and a simple Pauli-Sommerfeld behavior is found for $\Lambda^{\prime}$ :

$$
\Lambda^{\prime}=\ln \left(\frac{T^{(-)}}{T^{(+)}}\right)-\frac{\pi^{2}}{6}\left(\frac{k_{B} T}{\Gamma_{s}^{(+)}}\right)^{2} .
$$

Consideration of the obverse case $T^{(+)}>T^{(-)}$will yield similar results, but with an overall change of sign for $\Lambda^{\prime}$.

At any temperature, the $S$-shell impurity Green's function and thus also the $t$ matrix will show two resonances for each spin direction:

$$
\begin{aligned}
G_{d s}(\omega)= & \frac{1-\left\langle n_{d \vec{s}}\right\rangle}{\omega-\epsilon_{d s}-\delta E_{s}^{(-)}-i \Gamma_{s}^{(-)}} \\
& +\frac{\left\langle n_{d \vec{s}}\right\rangle}{\omega-\epsilon_{d s}-U-\delta E_{s}^{(+)}-i \Gamma_{s}^{(+)}} .
\end{aligned}
$$

The interplay between the two effects of the coupling of conduction electrons to the impurity, namely the lifetime broadening and the temperature-dependent shift of the ICEE's, can reproduce many of the experimental details associated with the dilute magnetic alloy problem.

Before we discuss the experimental effects for this simple $S$-shell model, it should be pointed out that for even more complex atoms we will expect that only two types of ICEE resonances will have energies near the Fermi energy and that several features of this simple model will carry over to more realistic models. The main import of our results is that the essential physics of the magnetic impurity is taking place primarily on the impurity site and that an understanding of the problem will require a determination of the impurity parameters: interconfigurational excitation energies (ICEE's) and the coupling parameters $V_{\overrightarrow{\mathbf{k}} d}$ for the many-electron states. In this paper, it can only be suggested a posteriori what values the parameters might have in order to reproduce magnetic impurity effects. The calculation of the parameters must await a procedure like Hirst's CRHF or something more sophisticated using many-electron stepping operators and many-electron state matrix elements.

The parameters which seem to give the best description of the various magnetic impurity effects are very simple. We need an effective $\Delta$ to be rather small, one of $\epsilon_{s}^{(r)}$ (measured relative to the Fermi energy) also small so that as $T \rightarrow 0$ the shift $\delta E_{s}^{(r)}$ tends to pull that ICEE resonance almost to the Fermi energy. When this happens the resistivity almost saturates, the heat capacity displays a very low- $T$ Schottky-like peak and the thermopower will show appropriate behavior.

A natural question which needs answering concerns the proposed pulling toward the Fermi energy of an ICEE: Why doesn't the resonance proceed on through zero, thus reversing the relative placement of the two relevant configuration energies, $E_{N}-N \mu$ and $E_{N+1}-(N+1) \mu$, measured with respect to the grand canonical ensemble? The answer is probably contained in the energetics of processes left out of the model. In particular, lattice energy associated with the different "size" of dif- 
ferent configurations can be of enough importance to force an impurity to retain the relative energy ranking of its configurations from high temperatures. This circumstance could possibly be reversed in the discussion of intermetallic compounds, where a change in the energy ordering of the whole lattice could be energetically favorable. The extension of this model to the resistivity ${ }^{59}$ of $\mathrm{CeAl}_{3}$ compounds appears to bear out these ideas. This will be discussed elsewhere.

Let us now proceed to examine the consequences of having one of the $E_{s}^{(r)}$ close enough to the Fermi energy that as $T \rightarrow 0$ the $\delta E_{s}^{(r)}$ shift pulls that ICEE down almost to the Fermi ene rgy。 In most of the following discussion I will consider $E_{s}^{(+)} \geqslant 0$ and small, while $E_{s}^{(-)} \ll 0$, and comment only briefly on the other case.

\section{A. Resistivity}

The temperature dependence of the impurity resistivity in our model reflects the movement through the Fermi energy of the leading half of an ICEE Lorentzian. Because the only change from this model which will transpire as we consider transition-metal atoms is the number of such resonance peaks at the Fermi energy, a direct comparison with the actual temperature dependence should be possible if the theory is properly scaled.

Let us first demonstrate that at high temperatures the leading temperature dependence of the resistivity is logarithmic as found by Kondo. ${ }^{60}$ This is easily done on realizing the dc resistivity is proportional to

$$
\pi N(0) \operatorname{Im} t=\sum_{s r} \frac{\left\langle N_{d s}^{(r)}\right\rangle \Gamma_{s}^{(r)} \Delta}{\left(E_{s}^{(r)}-\Delta \Lambda_{s}^{\prime} / \pi\right)^{2}+\left(\Gamma_{s}^{(r)}\right)^{2}} .
$$

At high temperatures, $\Lambda_{s}^{\prime}$ is small and proportional to $\ln \left(T /\left|\epsilon_{d s}\right|\right)$. Expanding the Lorentzian to leading order in small $\Lambda_{s}^{\prime}$ yields

$$
\begin{aligned}
\sum_{s r} \frac{\left\langle N_{d s}^{(r)}\right\rangle \Gamma_{s}^{(r)} \Delta}{\left(E_{s}^{(r)}\right)^{2}+\left(\Gamma_{s}^{(r)}\right)^{2}}\left[1+\frac{\Delta}{\pi} \frac{E_{s}^{(r)}}{\left(E_{s}^{(r)}\right)^{2}+\left(\Gamma_{s}^{(r)}\right)^{2}}\right. & \\
& \left.\times \ln \left(\frac{\left|\epsilon_{d s}\right|}{k_{B} T}\right)+\cdots\right]
\end{aligned}
$$

for the leading logarithmic dependence of the resistivity. Clearly, as the temperature decreases, such an expansion fails to hold and the logarithmic behavior is folded into the shape of the Lorentzian resonance. These considerations would suggest that it would be quite rare that an impurity would exhibit a strictly linear behavior in $-\ln T$ for very many decades of temperature. This is clearly in accord with the best experimental observations. ${ }^{61}$

It is worthwhile noting in passing that (4.31) recovers the behavior of the resistivity used in the pressure study of Sec. III.
A numerical solution of the thermodynamic selfconsistency equation for this model is used to generate the resistivity for parameters scaled to give a Kondo effect as we have been discussing. This calculation yields two characteristic temperatures of two types: the high-temperature $T^{(-)}$ $\approx\left|\epsilon_{d s}\right| / \pi$ which signals the onset (as $T$ decreases) of the high-temperature logarithmic behavior, and the low-temperature $T^{(+)} \approx \Gamma_{s}^{(+)} / \pi$ which gives the transition from $\Lambda^{\prime}$ logarithmic to the Sommerfeld low- $T$ behavior. For most alloys we may expect that $T^{(-)}$is large enough to be buried by the host phonon contributions to the high-temperature resistivity. Thus for most alloys $T^{(-)}$is basically unobservable. This leaves one parameter, $T^{(+)}$, which plays a role like that of the Kondo temperature. Low-temperature experiments should then give a universal curve (when scaled to some temperature $T^{(+)}$as a function of $T / T^{(+)}$. Figure 2 shows the comparison of the theoretical resistivity with the scaled resistivities of Loram et al. ${ }^{62}$ for $\mathrm{CuFe}$ systems. The most important feature of the agreement is not the high-temperature regime, but the excellent comparison in the intermediate range where $\Lambda^{\prime}$ is changing and where the peak of the Lorentzian is approaching the Fermi energy。 This is the first time a microscopic model which can be made compatible with the high-temperature Hund's rule spin values has shown such agreement with experiment in this range. ${ }^{63}$ Comparison of $T^{(+)}$of Fig。 2 and the Loram definition of $T_{\text {Kondo }}$ suggests that $T_{\mathrm{Kondo}} \approx 10 T^{(+)}$.

A short comment is perhaps appropriate here on the magnetoresistivity。 Examination of (4.31) shows that the resistivity is caused by both a spinup and a spin-down resonance at the Fermi energy. This may at first seem strange since the atomic configuration involved is a singlet $\left({ }^{1} S\right)$ state. It must be remembered that the resonance energies

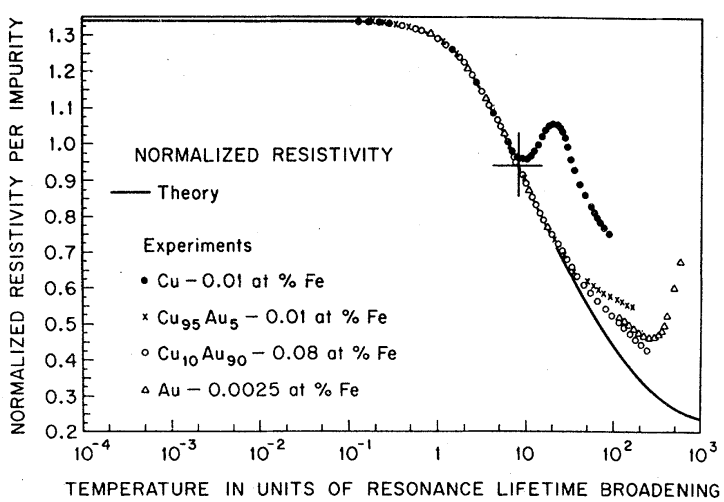

FIG. 2. Normalized resistivity of Loram et al. (Ref. 62) shown with the solid curve which is the theory of this paper. 
which appear in the $t$ matrix are the interconfigurational excitation energies, and the Zeeman energy of the level $e_{1 s}^{(+)}$arises from the $\left({ }^{2} S\right)$ energy in the difference

$$
E_{s}^{(+)}=E_{2}\left({ }^{1} S, 0\right)-E_{1}\left({ }^{2} S, m_{s}= \pm \frac{1}{2}\right) .
$$

The ICEE's can thus be split in a magnetic field and, as $T \rightarrow 0$, the resistivity measurements would observe the lower Zeeman-split energy resonance to pass through the Fermi energy; the resistivity then would have a maximum and saturate at a decreased value at $T=0$. This splitting out of the ICEE resonances at the Fermi surface well explains the negative magnetoresistivity associated with these impurities ${ }^{64}$ and well rationalizes the large-field behavior of several systems. If the ICEE is very broad, it may be difficult to resolve a peak in the resistivity, but for very narrow resonances $(\mathrm{Mn})$ this effect has been observed. ${ }^{65}$

Another effect of a magnetic field is to change the effective Kondo temperatures $T^{(+)}$at which the $\Lambda^{\prime}$ begins to approach a constant. The major consequence of this would be that the resonance energy $e_{s}^{(+)}$does not reach zero because the saturation value of $\Lambda^{\prime}$ [Eqs. (4.30) and (4.28)] is less than $\pi\left(\epsilon_{d s}+U\right) / \Delta$. In the situation where the magneticfield induced change of the effective temperature $T^{(+)}$dominates the Zeeman splitting of the resonance, a decrease in the resistivity with increasing field will be observed, and the "Kondo" temperature will behave like

$$
T_{K} \approx 10 T^{(+)} \approx \frac{10}{\pi}\left[\left(\mu_{B} H\right)^{2}+\left(\Gamma^{(+)}\right)^{2}\right]^{1 / 2} .
$$

The Daybell and Steyert ${ }^{66}$ data on $\mathrm{CuCr}$ showed that the temperature of the "knee" of the resistivity. shifted up with magnetic field in a fashion consistent with this expression.

In the low-temperature range the resistivity displays a Sommerfeld $T^{2}$ dependence, which arises not so much from the movement of the ICEE, but from the thermal population factors. This reflects the change in the population of the impurity levels as the coupled Fermi system approaches complete degeneracy. The low-temperature expression which which follows from Eq. (4.30) for this Anderson model is

$$
\frac{\rho}{\rho_{0}}=1-\left(\frac{k_{B} T}{3 \Gamma^{(+)}}\right)^{2}
$$

Because this expression reflects quite strongly the character of the $S$-shell model, comparison with experiments will not be made here. We just note that the temperature dependence is in accord with experiment for several systems. ${ }^{3}$
B. Thermoelectric power

The impurity thermopower $S_{\mathrm{th}}$ is most simply calculated using the simple expression given by MacDonald $^{67}$

$$
S_{\mathrm{th}}=\left.\frac{\pi^{2}}{3} \frac{k_{B}}{|e|}\left(k_{B} T\right) \frac{\partial}{\partial \omega} \ln \rho(\omega)\right|_{\omega=0}
$$

where $\rho(\omega)$ is the resistivity at energy $\omega$ from the Fermi energy. In contrast to the resistivity, the thermopower is partially dependent on the poles in the $t$ matrix which are not close to the Fermi energy; it is proportional to

$$
\frac{T}{\pi|\operatorname{Im} t|} \sum_{r s} \frac{\left\langle N_{d s}^{(r)}\right\rangle \Delta e_{2 s}^{(r)} e_{1 s}^{(r)}}{\left[\left(e_{1 s}^{(r)}\right)^{2}+\left(e_{2 s}^{(r)}\right)^{2}\right]^{2}}
$$

where the resonance parameters have been written in the notation of (4.23). For the low temperatures, the $e_{1 s}^{(+)}$are approaching zero, in the example being considered, while the $e_{1 s}^{(-)}$have not changed much from $\epsilon_{d s}$. The sign of the low-temperature thermopower will depend on the dominant contributions from the resonances which are near, but not closest to the Fermi energy.

For the sake of definiteness, let us examine the situation where $E_{s}^{(+)}$is positive and small, but $E_{s}^{(-)}$is negative and quite large. Then the $S$ shell impurity contribution to the thermopower is

$$
\begin{aligned}
S=\frac{\pi k_{B}^{2} T}{3|e||\operatorname{Im} t|}[ & \sum_{S}\left(\frac{\left(1-\left\langle n_{d \bar{s}}\right\rangle\right) \Delta^{2} E_{s}^{(-)}}{\left[\left(E_{s}^{(-)}\right)^{2}+\Delta^{2}\right]^{2}}\right) \\
& \left.+\frac{\left\langle n_{d \bar{s}^{-}} \Delta^{2}\left(E_{s}^{(+)}-\Delta_{\Lambda^{\prime}} / \pi\right)\right.}{\left[\Delta^{2}+\left(E_{s}^{(+)}-\Delta \Lambda^{\prime} / \pi\right)^{2}\right]^{2}}\right],
\end{aligned}
$$

where we have neglected $\Delta \Lambda^{\prime} / \pi$ compared to $E_{s}^{(-)}$. If $T^{(+)}<T<T^{(-)}$, the shift $\Lambda^{\prime}$ increases logarithmically as $T$ decreases and to lowest order in $E_{s}^{(+)}-\Delta \Lambda^{\prime} / \pi$ we may write, assuming $\left|E_{s}^{(-)}\right|>\Delta$,

$$
\begin{aligned}
S \propto \frac{T}{\Delta} & {\left[\left(1-\left\langle n_{d \bar{s}}\right\rangle\right)\left(\frac{\Delta}{E_{S}^{(-)}}\right)^{3}\right.} \\
& \left.+\left\langle n_{d \bar{s}}\right\rangle\left(\frac{E_{s}^{(+)}}{\Delta}-\frac{1}{\pi} \Lambda^{\prime}\right)+\cdots\right] .
\end{aligned}
$$

The impurity parameter values which give rise to a "Kondo" resistivity can concomitantly show a peak in the low-temperature thermopower, because as $T \rightarrow 0$ the second term in (4.36) goes almost to zero and the low-temperature $S$ will then have a negative linear slope in $T$. If $T>T^{(+)}$so $\Lambda^{\prime}$ varies like $\log (T)$, the temperature of the peak is given by

$$
\begin{aligned}
k_{B} T_{\text {peak }}=0.415\left|E_{s}^{(-)}\right| \exp \{ & -\pi\left[\frac{\left(1-\left\langle n_{d \bar{s}}\right\rangle\right)}{\left\langle n_{d \bar{s}}\right\rangle}\left(\frac{\Delta}{E_{s}^{(-)}}\right)^{3}\right. \\
+ & \left.\left.\frac{E_{s}^{(+)}}{\Delta}\right]\right\}
\end{aligned}
$$


Thus, if $T^{(+)}<T_{\text {peak }}$, a peak can occur in $S$. On the other hand if $T^{(+)}$is too large the low-temperature thermopower may appear to have no peak and may just be linear in the temperature. Because of the fact that the thermopower is dominated by the strongest scattering mechanism, phonon contributions may preclude experimentally seeing a peak if $T^{(+)}$is too large.

If we had considered the case $E^{(-)}$small and $E^{(+)}$large, we would have found similar behavior but with a change of sign. Remember that $\Lambda^{\prime}$ has an overall sign change in this case and also, the thermopower will reflect another change of sign due to the $(+)$ resonances far from the Fermi energy contributing a positive term to $S$. The sign of the low-temperature $d S / d T$ in this theory is dependent upon impurity configuration energy spacing. If $E^{(+)}$is small then $d S / d T<0$ as $T \rightarrow 0$. Or if $E^{(-)}$is small, then $d S / d T>0$ as $T \rightarrow 0$.

The sign of $S$ at low temperatures is dependent upon the nature of the interconfigurational excitation energies of the impurity in the host. As will be discussed briefly below, if this picture is correct then the predominance of negative $S$ for the iron-group atom $\mathrm{s}^{68}$ suggests the importance of $d^{n-1} s^{2} \rightarrow d^{n} s^{1}$ core to valence transitions in these impurities as opposed to actual ionization of the impurity site. ${ }^{69}$ This line of discussion suggests that, like many other properties of the magnetic impurity problem in this theory, many-electron properties of the impurity dominate the system's reponse to external probes. (A calculation of $S$ is shown in Fig. 3.)

\section{Zero-field susceptibility}

The expression for the zero-field magnetic susceptibility of the impurity is given even in the interacting case by the same expression (3.24) as

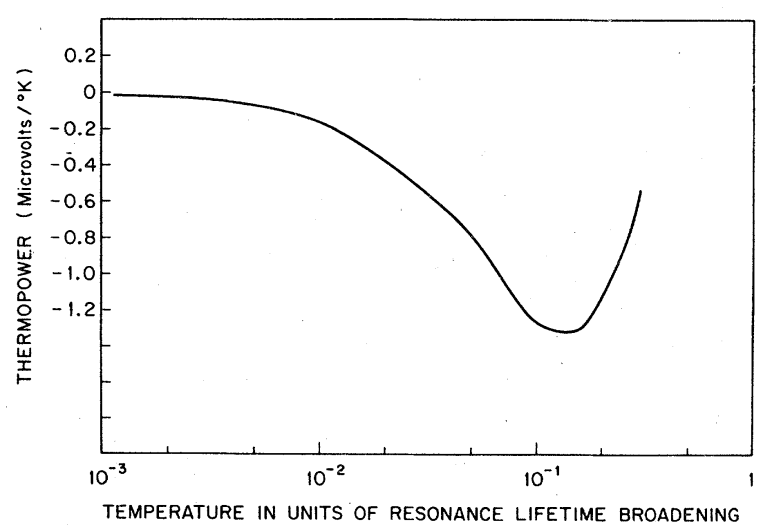

FIG. 3. This figure shows the impurity contribution to the thermopower for the same theoretical parameters which gave a good fit to the resistivity in Fig. 1. for the free atom, except that the expression for

$$
f_{s}^{(r)}=\left\langle\phi_{s}^{\dagger}(r) \phi_{s}^{(r)}\right\rangle
$$

must reflect the coupled system. By examining the expressions for these thermal averages we find from Appendices $\mathrm{A}$ and $\mathrm{C}$ that

$$
f_{s}^{(r)}=\frac{1}{2}-\frac{1}{\pi} \operatorname{Im} \psi\left(\frac{1}{2}+\frac{\beta e_{2 s}^{(r)}}{2 \pi}+\frac{i \beta e_{1 s}^{(r)}}{2 \pi}\right),
$$

where $\psi$ is the complex digamma function, $\beta$ $=1 / k_{B} T$ and $e_{1 s}^{(r)}$ and $e_{2 s}^{(r)}$ are the real and imaginary part of the Green's function pole corresponding to the $r$ th ICEE.

The temperature dependence of the impurity susceptibility will then be determined by the behavior of the $f_{s}^{(r)}$. For an impurity coupled to a conduction-electron gas with a constant density of states at the Fermi surface, the temperature dependence is determined by the degeneracy temperature $T^{(r)}$ associated with the $r$ th ICEE. If $T>T^{(r)}$, then

$$
-\frac{\partial f_{s}^{(r)}}{\partial e_{1 s}^{(r)}} \approx \frac{0.204}{k_{B} T}+O\left(\frac{1}{T^{2}}\right),
$$

so at high temperatures the susceptibility can be Curie-like. However, if $T<T^{(r)}$, the temperature dependence for the $f_{s}^{(r)}$ becomes characteristic of a degenerate Fermi system. As is shown in Appendix $\mathrm{C}$ for $T<T^{(r)}$,

$$
-\frac{\partial f_{s}^{(r)}}{\partial e_{1 s}^{(r)}}=\frac{1}{\pi} \frac{e_{2 s}^{(r)}}{\left(e_{1 s}^{(r)}\right)^{2}+\left(e_{2 s}^{(r)}\right)^{2}}-O\left(T^{2}\right) .
$$

The temperature behavior of the impurity susceptibility at lower temperatures is dominated by the smallest $T^{(r)}$. For temperatures larger than this $T^{(r)}$ the susceptibility will display a CurieWeiss behavior, but at temperatures comparable to or lower than $T^{(r)}$ the susceptibility will appear to be more Pauli-like.

The physical basis of this behavior shows some similarities to the fluctuation picture presented by Wohlleben and Coles $^{70}$ and others. ${ }^{71}$ In fact, the average fluctuation frequency given by

$$
\hbar \omega_{f}=k_{B} T_{f}
$$

in this picture plays exactly the role of the smallest temperature $T^{(r)}$ here: if the temperatures are high compared to the energy $k_{B} T^{(r)}$, the occupation densities of the magnetic sublevels of the impurity can be significantly altered by the external magnetic field. In this temperature range there is sufficient thermal energy so that the magnetic degrees of freedom of the impurity are more or less uncoupled from the conduction-electron system. Thus the moment fluctuations resemble an almost free impurity with its characteristic Curie susceptibility. If the temperature 
is low or comparable to $T^{(r)}$, the degeneracy of the conduction-electron system begins to dominate the impurity system's magnetic response. Now the coupled composite many-particle states comprised of both impurity and host electrons become dominated by the density of states of the coupled system. Effectively, for $T<T^{(r)}$, the impurity cannot change its spin independently of the many conduction electrons to which it is coupled. The spin fluctuations are dominated by the density of states of the coupled states and appear to be Paulilike in their behavior. Thus, just as in the freeelectron $^{72}$ case, the new ground state in the presence of a magnetic field, and the states just above the ground state in energy, all have moments parallel to the field. The antiparallel moment states are much higher in energy compared to $k T$. Such a distribution of many-electron states gives rise to a susceptibility that reflects the moments of the ground state and its nearby states and is then effectively a Pauli susceptibility .

Let us now examine briefly the analytic susseptibility results of the $S$-shell Anderson model to see if there are any consequences of general application The first such result occurs for the high-temperature susceptibility. It has been observed experimentally ${ }^{73}$ that the high-temperature Curie-Weiss susceptibility of iron-group impurities gives a somewhat reduced effective moment when compared to the atomic moment. It is of immediate interest to note that precisely that condition which gives the characteristic resistivity of such magnetic impurities, namely, that one of the ICEE resonances is close to the Fermi energy and is pulled toward the Fermi energy by the coupling, contributes to a reduced moment. In the $S$-shell model the reduction in the moment comes about because of the change in the weighting of the different impurity configurations due to the conduction-electron coupling.

Second, the $S$-shell model spin susceptibility at high temperatures is determined from the high temperature expansion of the digamma function:

$$
\chi=\frac{0.5403 \mu_{B}^{2}}{k_{B} T}\left(1-\frac{\theta_{\chi}}{T}\right),
$$

where

$$
\theta_{\mathrm{x}}=0.675 \Gamma_{s}^{(+)}
$$

where $\Gamma_{s}^{(+)}$is the linewidth of the ICEE and $E_{s}^{(+)}$ is the high-temperature energy of the (+) ICEE. Note first that for this model in the atomic limit the susceptibility is $\chi / \mu_{B}^{2}=1 / k_{B} T$, so that the numerator in (4.42) is the effective moment squared in Bohr magnetons. This yields a ratio for the effective moment to the Hund's rule moment of
0.739 , which is very close to the CuMn ratio of $0.75 \pm 0.05 .^{74}$ Further comparisons yield reasonable agreement with atoms across the iron group. The "Weiss" temperature $\theta_{x}$ is positive unless $E_{s}^{(+)}$becomes too large. However, estimates of the values of $E_{s}^{(+)}$which will give a Kondo resistivity and bring the ICEE resonance near the Fermi energy as $T \rightarrow 0$, that is

$$
\frac{E_{s}^{(+)}}{\Gamma_{s}^{(+)}} \approx \frac{1}{2 \pi} \ln \left[\frac{\left(E_{s}^{(-)}\right)^{2}+\left(\Gamma_{s}^{(-)}\right)^{2}}{\left(E_{s}^{(+)}\right)^{2}+\left(\Gamma_{s}^{(+)}\right)^{2}}\right] .
$$

suggests that $\theta_{x}$ is always positive, though possibly less than characteristic temperatures measured in other effects.

The low-temperature analysis for the susceptibility, which gives the susceptibility in the degenerate Pauli region, yields

$$
\frac{\chi}{\mu_{B}^{2}}=\frac{8}{3 \pi} \frac{\xi^{(+)}}{\Gamma_{s}^{(+)}}\left[1-\frac{\pi^{2}}{3}\left(\frac{k_{B} T}{\Gamma_{s}^{(+)}}\right)^{2}+\cdots\right],
$$

where $\xi^{(+)}$is a thermal weighting factor

$$
\xi^{(+)}=\frac{f_{0}^{(-)}}{1+\frac{4}{3} f_{0}^{(-)}\left(1-f_{0}^{(-)}\right),},
$$

which approaches 1 as $f_{0}^{(-)} \rightarrow 1$. This form of the susceptibility is calculated assuming the $e^{(+)}$ resonance approaches the Fermi energy and that $k_{B} T<\Gamma_{s}^{(+)} / \pi$. This form is very close to the spin fluctuation results for low temperatures. ${ }^{75}$.

The susceptibility has also been calculated $\mathrm{nu}$ merically for the whole temperature range in a normalized form and Figs 4 and 5 show the results.

At this point a brief comparison of these results with the standard perturbative result on the susceptibility by Scalapino ${ }^{76}$ may be employed to show a possible relationship between this work

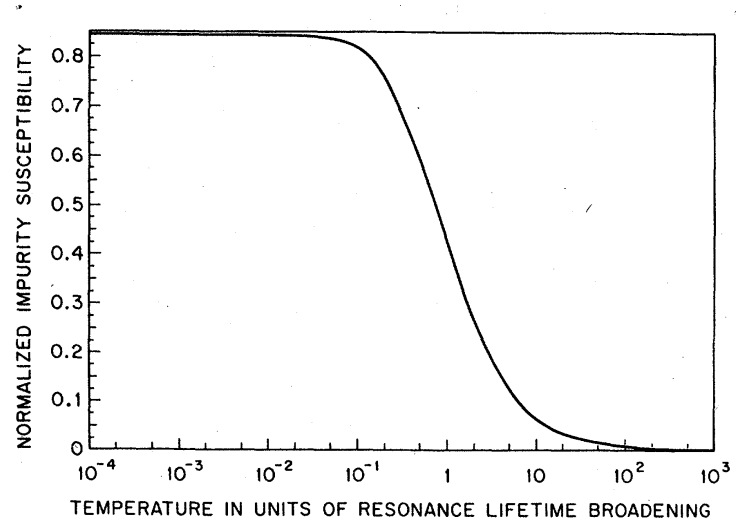

FIG. 4. This figure shows the normalized impurity susceptibility plotted against temperature which is normalized to the ICEE lifetime broadening, for the same parameters as in Fig. 1. 
and other more traditional approaches. We will show that it is possible to reproduce Scalapino's results in an asymptotic expansion to logarithmic accuracy, but a more careful examination will show that this expansion is not physical for Scalapino's choice of parameter values. Also, the same $J_{\text {eff }}^{\prime}$ as was derived for the resistivity will be recovered.

Scalapino used the standard thermodynamic perturbation expansion for the partition function, calculated an expansion for the free energy, and evaluated the impurity susceptibility exactly to second order in the coupling $V$; and to logarithmic accuracy in fourth order. The result of this evaluation was

$$
\chi_{\text {imp }}=\frac{\mu_{B}^{2}}{k_{B} T}\left\{1+N(0) J\left[1+N(0) J \ln \left(\frac{D}{k_{B} T}\right)\right]+\cdots\right\} .
$$

To recover this expansion here it is necessary to evaluate our expression for $\chi^{\text {imp }}$ (3.24) using an approximation for $f_{s}^{(r)}$. To do this we seek an expansion in the lifetime broadening of the ICEE resonance, keeping the shift of the real part of the energy in the Fermi function. The appropriate expansion is

$$
\begin{aligned}
f_{s}^{(r)}= & \frac{1}{e^{\beta E_{s}^{(r)}}+1}+\frac{\Gamma_{s}^{(r)}}{\pi E_{s}^{(r)}}+\frac{k_{B} T}{E_{s}^{(r)}}\left(\frac{\Gamma_{s}^{(r)}}{E_{s}^{(r)}}\right)^{2} \\
& -\frac{4 \pi}{3}\left(\frac{k_{B} T}{E_{s}^{(r)}}\right)^{2} \frac{\Gamma_{s}^{(r)}}{E_{s}^{(r)}},
\end{aligned}
$$

where $E_{s}^{(r)}$ includes the shift (4.24) and $\Gamma_{s}^{(r)}$ is the lifetime broadening of the resonance. This expression is valid if $\Gamma_{s}^{(r)} \ll\left|E_{s}^{(r)}\right|$ and $k T \ll\left|E_{s}^{(r)}\right|$. To recover Scalapino's result we must keep only the Fermi function term and neglect the other terms. For accurate numerical approximations this is not a consistent scheme since the second term in the expansion dominates the first for some energy and temperature ranges. This is particularly true if $T<T^{(r)}$. Remembering that

$$
e_{1 s}^{(r)}=\epsilon_{d s}+\frac{1}{2} U(1+r)-\delta E_{s}^{(r)},
$$

it is straightforward to show that, in the same regime $\left(\epsilon_{d s}<0, \epsilon_{d s}+U>0, T<T^{(r)}\right)$ that Scalapino studied,

$$
\chi_{\mathrm{imp}}=\frac{\mu_{B}^{2}}{k_{B} T}\left[1+\frac{\partial}{\partial H}\left(\delta E_{s}^{(+)}\right)\right]
$$

where in deriving (4.49) the fact has been used that, to lowest order in the magnetic field, $\delta E_{s}^{(r)}=\delta E_{s}^{(-)}$. Using the properties of the $s_{D}$ functions and $\delta E_{s}^{(r)}$ from the Appendices, we can show that

$$
\begin{aligned}
\frac{\partial}{\partial H}\left(\delta E_{s}^{(+)}\right)=\frac{\Delta}{\pi\left\langle n_{d \bar{s}}\right\rangle} & \left(\left.\left\langle n_{d s}\right\rangle \frac{\partial \boldsymbol{S}_{D}^{\prime}}{\partial E}\right|_{E_{s}^{(+)}-\delta E_{s}^{(+)}}\right. \\
& -\left.\left(1-\left\langle n_{d s}\right\rangle\right) \frac{\partial \mathcal{G}_{D}^{\prime}}{\partial E}\right|_{E_{s}^{(-)}-\delta E_{s}^{(-)}} \\
& -2\left(1-\left\langle n_{d \vec{s}}\right\rangle-\left\langle n_{d s}\right\rangle\right) \\
& \left.\times\left.\frac{\partial \mathcal{S}_{D^{\prime}}}{\partial E}\right|_{-E_{s}^{(-)}-6 E_{s}^{(+)}}\right)
\end{aligned}
$$

For the parameter range under study the last term vanishes, and

$$
\frac{\partial \mathfrak{S}_{D}^{\prime}(E, \Gamma)}{\partial E}=\frac{E+D}{(E+D)^{2}+\Gamma^{2}}-\frac{E}{E^{2}+\Gamma^{2}}+O\left(T^{2}\right) .
$$

Neglecting the $\Gamma^{2}$ compared to the $\epsilon$ and $\epsilon+D$ we find for the susceptibility

$$
\begin{aligned}
\chi_{\mathrm{Imp}}=\frac{\mu_{B}^{2}}{k_{B} T}\left[1+\frac{\Delta}{\pi}\right. & \left(\frac{1}{\epsilon_{d s}-\delta E_{s}^{(-)}}-\frac{1}{\epsilon_{d s}+U-\delta E_{s}^{(+)}}\right. \\
& +\frac{1}{D+\epsilon_{d s}+U-\delta E_{s}^{(+)}} \\
& \left.\left.-\frac{1}{D+\epsilon_{d s}-\delta E_{s}^{(-)}}\right)+\cdots\right]
\end{aligned}
$$

The zeroth-order expansion of this second term is Scalapino's first term

$$
N(0) J=V_{\mathrm{k} d}^{2} N(0)\left(\frac{1}{\epsilon_{d s}}-\frac{1}{\epsilon_{d s}+U}\right) .
$$

The first-order expansion of the denominators $\delta E_{s}^{(r)}$ yields for the next term a term

$$
[N(0) J]^{2}\left(1+\frac{2 \epsilon_{d s}}{U}\right) \Lambda^{\prime}
$$

if we insist on writing expressions in terms of the effective $J$ of (4.53). If one of the resonances dominates the shift as in the resistivity, the same effective $J$ as found in Sec. III is recovered here. Now if one seeks to recover only the $\log (T)$ part of $\Lambda^{\prime}$ without regard for the fact that $\Lambda^{\prime}$ depends on $\Gamma$, and only displays this behavior for $T^{(-r)}$ $<T<T^{(r)}$, then one achieves a $\log (T)$ term in the expansion for the susceptibility. However, it is well known that perturbation expansions are often asymptotic and, when only a particular functional form of an expansion $[\log (T)]$ is sought, there is little control of the region of convergence or applicability. As illustrated above there is a regime within which an asymptotic expansion of Scalapino's form can be found for $\chi_{1 \text { mp. }}$. However, there are at least two grounds on which it can be argued that this expansion will not be seen experimentally. First, the expansion for $f_{s}^{(r)}$ is dom- 
inated by the Pauli terms $(\Gamma / E)$ instead of the Fermi function first term. Second, the logarithmic behavior of $\Lambda^{\prime}$ will not appear in the same range of temperatures as does the range in which Scalapino's first term is found. The actual temperature dependence of the susceptibility is controlled rather more strongly by the degeneracy temperatures $T^{(r)}$, one of which is dominated by the ICEE broadening if $E_{s}^{(r)}$ is small. The susceptibility displays the transition from a Curie behavior to a degenerate Pauli susceptibility which depends more on the lifetime broadening than on the shift of the ICEE. It is possible for impurity parameters of the sort which give a "Kondo" effect and ICEE fluctuations that $\ln T$ behavior at high temperatures can be seen. The major point is that coefficients of the expansion are different from those which the usual theoretical treatments would predict.

\section{Heat capacity}

The heat capacity of the impurity-host system which shows the "Kondo" resistivity should also show what is effectively a Schottky anomaly at low temperatures. This interpretation cannot be taken too literally since the energies of the impurity resonances are themselves temperature dependent. However, the heat capacity will show a peak at low temperatures.

The heat capacity is calculated using

$$
C_{V}=C_{V}^{\text {atomic }}+C_{V}^{\text {int }} \text {, }
$$

where $C_{V}$ atomic can be expressed in terms of $f_{s}^{(r)}$ functions, giving a formula like the atomic limit, but calculated with the fully interacting $f_{s}^{(r)}$. The second term is

$$
\begin{aligned}
C_{V}^{\mathrm{int}}=-\frac{2 \Delta}{\pi} \sum_{s} & \left(1-\left\langle n_{d \vec{s}}\right\rangle\right) \frac{\partial \lambda_{\bar{s}}^{(-)}}{\partial T}+\left\langle n_{d \vec{s}}\right\rangle \frac{\partial \lambda_{\bar{s}}^{(+)}}{\partial T} \\
& \left.+\Lambda_{s}^{\prime} \frac{\partial\left\langle n_{d \vec{s}}\right\rangle}{\partial T}\right) .
\end{aligned}
$$

When the interacting system $f_{s}^{(r)}$ are evaluated the low-temperature behavior of $C_{V}$ is dominated by $C_{V}^{\text {int }}$ and shows a peak around the smallest $T^{(r)}$. If the $T^{(r)}$ is small enough to give the "Kondo" resistivity, this same condition gives rise to a low $T$ peak in the heat capacity. In Fig. 6 is a plot of the heat capacity calculated for a set of parameters which closely approximates the experimental heat capacity of $\mathrm{La}_{1-x} \mathrm{Ce}_{x}$ alloys as measured by Bader and Phillips. ${ }^{77}$ For this calculation, the theoretical parameters have been fitted to give the experimental temperature scale, and the magnitude of the heat capacity was multiplied by a factor of 0.88 to bring the magnitude of the theory into accord with experiment. This factor

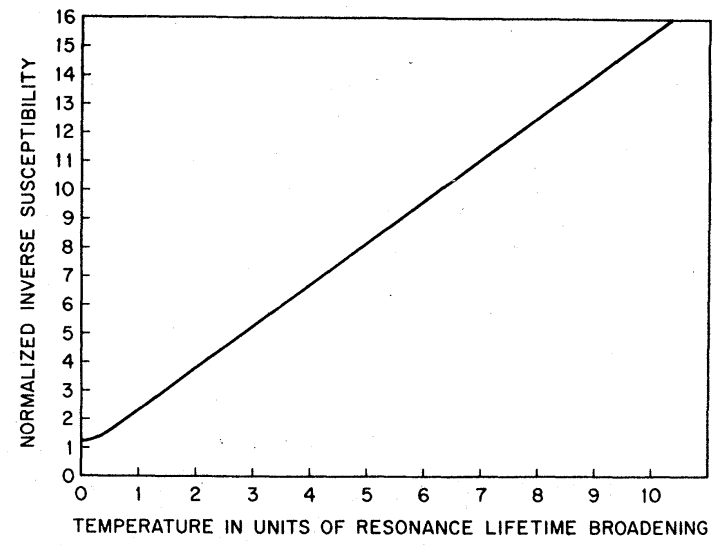

FIG. 5. This figure shows the normalized inverse susceptibility for the same parameters as in Fig. 1.

would correspond to a 0.08 -at. \% error in the experimental concentration of impurities in the alloy. While this possibility has not been explored in detail for this system, such an error is not unreasonable for heat capacity measurements on impurities. ${ }^{78}$

It is of interest to note that the best theoretical fit to the low $-T$ data suggests that the second peak in the heat capacity due to the other ICEE is close enough to the lowest one that it may have already been seen exper imentally (see Fig. 6). Further study of this possibility should be made in the future.

At this point we give a brief comparison of the low-temperature ratio $C_{V} / T \chi$ calculated in this theory and in Wilson's calculation of the spin $-\frac{1}{2}$ Kondo model. Using the expressions (4.56) and (3.24) for $C_{V}$ and $\chi$, it can be shown that in the strong coupling limit ( $\Delta$ dominating all other quantities)

$$
\frac{\mu_{B}^{2} C_{Y}}{T \chi} \rightarrow \frac{4 \pi^{2}}{3}
$$

in agreement with Wilson. ${ }^{79}$ On the other hand, if the model parameters are fixed so that the $(+)$ resonance approaches the Fermi energy as $T \rightarrow 0$, which condition gives the "Kondo" effect in this paper, the ratio gives exactly the same value found by Wilson, ${ }^{79} \frac{2}{3} \pi^{2}$ for the Kondo Hamiltonian. This result obtains from the following low-temperature expressions

$$
\frac{C_{V}}{k_{B} T}=\frac{8 \pi}{9 \Delta}+\frac{4 \pi}{9 \Delta} \frac{y^{4}}{\left(1+y^{2}\right)^{2}}-\frac{5 \pi}{27 \Delta} \frac{y^{2}}{\left(1+y^{2}\right)^{2}}
$$

and

$$
\chi=\frac{4 \mu_{B}^{2}}{3 \pi \Delta}\left(1+\frac{y^{2}}{1+y^{2}}\right),
$$

where $y=\Delta / \epsilon$ and is very small. Neglecting the $y$ terms yields the desired result. 


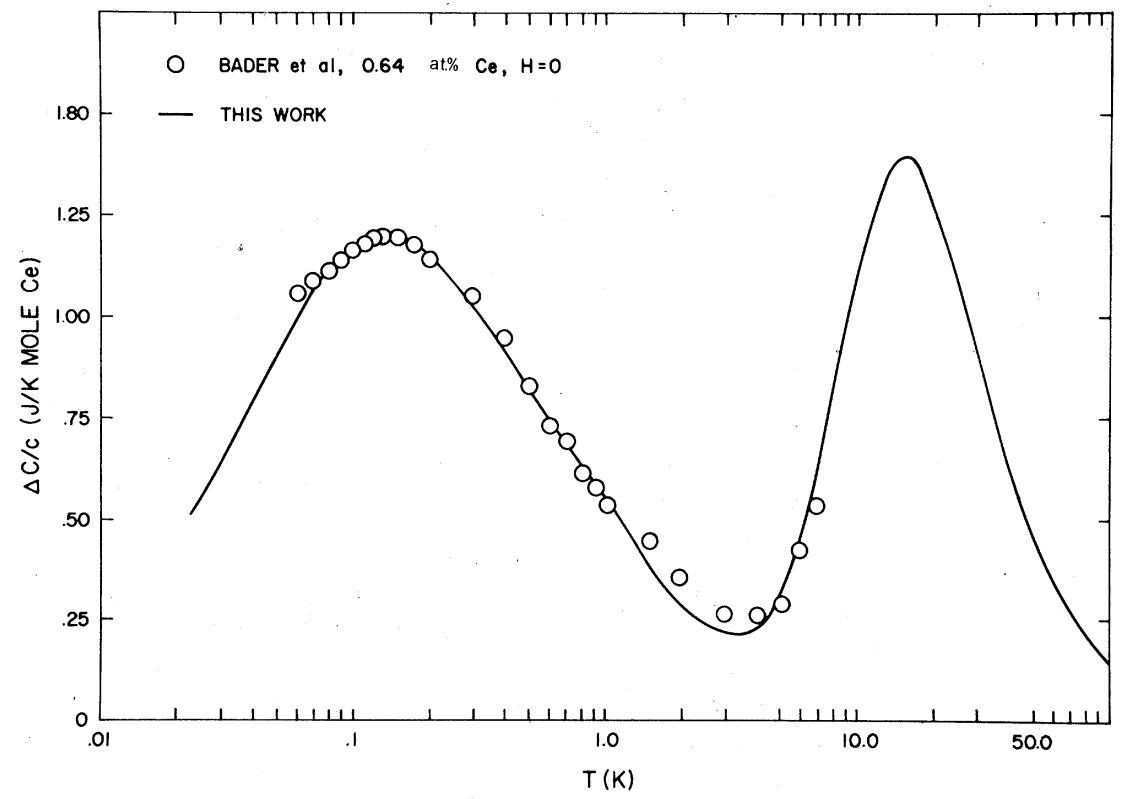

FIG. 6. This figure shows the fit of the theoretical heat capacity with a temperature scale chosen to fit the experimental temperatures. See text for full explanation.
In another paper, we examine a comparison of the calculated values of $T \chi$ for the Krishnamurty, Wilkins, Wilson ${ }^{80}(\mathrm{KWW})$ method and the calculations of this paper. In general the high-temperature and low-temperature results agree, but a substantial difference occurs in the temperature range in which the susceptibility changes from CurieWeiss to Pauli-like. The KWW result gives the change over at consistently lower temperatures for the same parameters than do the calculations of this work. While the two methods are not directly comparable, the major difference seems to be that the KWW density of states for the conduction electrons has a logarithmic peak at the Fermi energy. The calculations of this work employ a constant conduction electron density of states near the Fermi energy. The difference between these two methods will be discussed in more detail elsewhere. ${ }^{81}$

\section{TRANSITION-METAL IMPURITIES IN METALS}

In this section, the many-electron extension of the results of the simple $S$-shell calculation and other implications for the impurity with a partially filled $d$ or $f$ shell will be briefly and qualitatively discussed. Most of the emphasis will be placed on the iron group, though the explanations are quite general.

The first element of unfinished business is the question of the lifetime broadening of the ICEE's. Perhaps the easiest way to approach this is to begin with Hirst's equation of motion of the conduction electrons. Written in our operator lan- guage this equation is

$$
\dot{L} c_{\overrightarrow{\mathrm{k}} s}=\epsilon_{\overrightarrow{\mathrm{k}} s} c_{\mathrm{k} s}+\sum_{m} V_{\overrightarrow{\mathrm{k}} m s} c_{m s},
$$

where $m=-l, \ldots,+l$ and $s= \pm \frac{1}{2}$ label the orbitals of the impurity shell. There are two comments which are relevant here. First, the $V_{\vec{k}} m s$ arise as variational parameters in the CRHF-like procedure. It is especially important not to attempt to evaluate these coupling parameters using results of calculations which do not fully consider the many-electron configurations of the impurity.

Second, it is probably necessary to redo Hirst's formulation of this problem so that instead of (5.1) an equation of motion involving the Judd stepping operators $\phi_{n}(\alpha, \beta)$ is determined by some variational principle analogous to Hirst's CRHF. This would probably yield instead of (5.1), an equation of the form

$$
L c_{\overrightarrow{\mathrm{k}} s}=\epsilon_{\overrightarrow{\mathrm{k}} s} c_{\overrightarrow{\mathrm{k}} s}+\sum_{r \mid \alpha \beta} V_{\overrightarrow{\mathrm{k}} \alpha \beta n}^{l} \phi_{n}(\alpha, \beta) .
$$

In the comparisons with experiment the small size of the ICEE widths found suggests that manyelectron matrix elements like $V_{\mathrm{k} \alpha \beta n}^{\ell}$ should be used in place of the CRHF one-electron variational parameters $V_{\overrightarrow{\mathrm{k}} m s}$. The estimation of these manyelectron matrix elements requires a more sophisticated reexamination of the $(n-1, n, n+1)$ electron impurity Hamiltonian in a variational scheme which more carefully treats the manyelectron states.

Short of pursuing such a fundamental approach at this time, a relatively crude estimate of the 
effective line width variation across a family like the $3 d$ family will be attempted. The main idea is that the effective matrix element in the Green's function is

$$
V_{\vec{k} \alpha \beta n}^{\text {eff }}=V_{\overrightarrow{\mathrm{k}} \alpha \beta n}\left\|\tilde{\phi}_{n}(\alpha, \beta)\right\| \text {. }
$$

The variation of the linewidth across a group like the $3 d$ 's will reflect the variation of

$$
\Gamma_{\alpha \beta n}=N(0) V_{\mathrm{k} \alpha \beta n}^{2}\left\|\tilde{\phi}_{n}(\alpha, \beta)\right\|^{2},
$$

as $n$ varies from $n=1$ to $n=10$. The simplest estimate of

$$
\left|a_{\alpha \beta n}^{m s}\right|^{2} \equiv\left\|\tilde{\phi}_{n}(\alpha, \beta)\right\|^{2},
$$

can be obtained by assuming that both the $\alpha$ and $\beta$ states are single determinants. Then the form $\tilde{\phi}_{n_{0}}(\alpha, \beta)$ would be

$$
\tilde{\phi}_{n_{0}}(\alpha, \beta) \approx c_{\alpha_{1}}^{\dagger} \ldots c_{\alpha_{n_{0}-1}}^{\dagger} I_{\theta} c_{\beta_{1}} \ldots c_{\beta_{n_{0}}},
$$

where the $\alpha_{i}$ and $\beta_{i}$ label the $m_{i} s_{i}$ in each determinant. Recalling from Sec. III that $I_{\theta}$ is a product of all $c c^{\dagger}$ for all states of the shell, a calculation of $|a|^{2}$ yields, for a $d$ shell, the expectation of $n_{0}-1$ factors of $n_{\alpha}$ and $9-n_{0}$ factors of $1-n_{\alpha}$. The most naive estimate of this is to replace each $n_{\alpha}$ by the fractional occupation $0 \leqslant x$ $\leqslant 1$ of each orbital. Then, we estimate $|a|^{2}$ to be proportional to

$$
(x)^{n_{0}-1}(1-x)^{9-n_{0}} \text {. }
$$

This dependence gives relatively narrower ICEE linewidths for the resonances near the Fermi surface as one passes through the iron-group family. Thus, this estimate would make the $n_{0}=1, x=0.1$ and the $n_{0}=5, x=0.5$ linewidths compare as the ratio of about $1: 10^{-3}$.

The preceeding estimate can be evaluated much more precisely by using the true correlated combinations of products of creation operators which create the terms of the shell. These linear combinations can be obtained from standard tables such as Slater. ${ }^{82}$ The coefficients $a$ can be expanded in terms of Wigner 3-j symbols, Racah's coefficients of fractional parentage ${ }^{83}$ and thermal expectation values of polynomials on one-electron number operators. These expectation values must be in principle evaluated in the grand canonical ensemble or equivalently as solutions of thermodynamic self-consistency equations in a Green's function calculation.

Even with the exact expressions for the expansion parameters, it is difficult at this time to improve much on the estimates above. The lifetime broadening of an ICEE could be much smaller than any one electron estimate, and we should expect that impurities with half-filled or close to half-filled shells will have much narrower ICEE's than atoms on either end of the series.

The major implication of our study to this point is that the presence of impurity ICEE's near the host Fermi energy is what most strongly influences the alloy properties. The equation of motion of each $\phi_{n}(\alpha, \beta)$ may be manipulated exactly as in the simple $S$-shell model. There will be the diagonal terms and the mixing terms. The thermal shift of the ICEE energies will arise in the same fashion as before, though now there will be many more sets of terms. The essential effects, however, will arise from those ICEE's which are nearest to the Fermi surface. One might expect only two types of ICEE's to be important for a many-electron atom. This suggests that the major difference between a realistic impurity and the $S$-shell model is a matter of how many of each type of ICEE there are. Many of the simplest properties of this model should be applicable.

Thus, for every alloy there will be some smallest effective degeneracy temperature

$$
T_{E, \Gamma}=\frac{1}{2 \pi}\left(E^{2}+\Gamma^{2}\right)^{1 / 2},
$$

which will determine the changeover from a logarithmic ICEE shift and a Curie-like susceptibility to a Sommerfeld $1-T^{2}$ shift and a Pauli-like susceptibility.

To illustrate one application of these ideas, the variation of the resistivity through the iron group will be briefly examined. As is well known, ${ }^{84}$ at low temperatures this quantity exhibits a single peak at about $\mathrm{Cr}$ or $\mathrm{Mn}$, which peak in the traditional Hartree-Fock ${ }^{85}$ picture is represented by a $\sin ^{2}\left(\frac{1}{10} \pi N\right)$ functional form. At an elevated temperature the resistivity of those elements in the center of the family $(\mathrm{Cr}, \mathrm{Mn}, \mathrm{Fe})$ is less than the "outer" members of the family. In many early treatments ${ }^{86}$ of this problem this behavior was regarded as experimental verification of Anderson's HF treatment of this model. In what follows, we will examine the explanation of these results from the perspective of this study, using the result that an ICEE is near to and pulled toward the Fermi energy by the conductionelectron coupling and that the ICEE linewidth is smallest at the center of the family.

Let us first examine the low-temperature resistivity. If every impurity at low temperatures fits into the picture I have been presenting here, then the resistivity should be simply a measure of the number of interconfigurational channels from $n$ electrons to $n+1$ electrons which are saturated for that specie of impurity. If one assumes a Hund's rule ground state for the impurities, it is easy to show that $N_{n, n+1}$, the num- 
ber of channels between $d^{n} \rightarrow d^{n+1}$, is simply proportional to Schrieffer's result which has the residual resistivity proportional to $2 S_{n}$, where $S_{n}$ is the Hund's rule spin of the $d^{n}$ ground state. However, as noted by Schrieffer ${ }^{87}$ and others the experimental data on residual resistivities is really not good enough to distinguish between the Schreiffer result and the HF result. Furthermore, as will be discussed below some of the impurities at the ends of the family may not have reached the unitarity limit at low $T$ and this could decrease their residual resistivities below the straight lines of our Schrieffer-like prediction. This last effect would appear to be approximated by the HF $\sin ^{2}\left(\frac{1}{10} \pi n\right)$ result and thus lessen the value of residual resistivities as a distinguishing experiment.

Let us now qualitatively examine the finite-temperature behavior of the resistivity. The impurity resistivity will be given roughly by

$$
\rho \propto \frac{N_{n, n+1} \Gamma_{n}^{2}}{\Gamma_{n}^{2}+\left(\epsilon_{n}-\Lambda_{n}\right)^{2}},
$$

where $N_{n, n+1}$ gives the number of channels, $\Gamma_{n}$ is the effective width of the important ICEE, $\epsilon_{n}$ is the ICEE, and $\Lambda_{n}$ the shift we have been studying. [We are assuming that at low temperatures the ICEE value $\Delta \epsilon_{0}$ given by

$$
\Delta \epsilon_{0}=\epsilon_{n}-\Lambda_{n}=\epsilon_{n}-\frac{\Gamma_{n}}{\pi} \ln \left(\frac{T_{n}^{(-)}}{T_{n}^{(+)}}\right)
$$

is a small quantity.] The low-temperature resistivity will be

$$
\rho \propto \frac{N_{n, n+1} \Gamma_{n}^{2}}{\Gamma_{n}^{2}+\left[\epsilon_{n}-\frac{\Gamma_{n}}{\pi} \ln \left(\frac{T_{n}^{(-)}}{T_{n}^{(+)}}\right)\right]^{2}} .
$$

If $\Delta \epsilon_{0}=0$ we achieve the unitarity limit. If the temperature increases to $T>T_{n}^{(+)}$, then we can write the resistivity as

$$
\rho \propto \frac{N_{n, n+1}}{1+\left[\frac{\Delta \epsilon_{0}}{\Gamma_{n}}-\frac{1}{\pi} \ln \left(\frac{T}{T_{n}^{(+)}}\right)\right]^{2}} .
$$

The amount by which the resistivity will have decreased at this elevated temperature will depend on both $\Delta \epsilon_{0}$ and $T_{n}^{(+)}$. We examine some relevant values of these quantities. First, if $\Delta \epsilon_{0}=0$ and $T_{n}^{(+)}$is quite small, the impurity could have both achieved the unitarity limit at $T \simeq 0$ and exhibited a significant decrease for $T>T_{n}^{(+)}$. From the perspective of this study, this case would seem to apply to impurities near the center of the iron group. A case which appears to be more applicable to impurities away from the center of the group corresponds to $\Delta \epsilon_{0} \neq 0$. In this case, two effects are present which imply that the decrease of the resistivity at the temperature $T$ will be less than that in the just preceeding case. First, since $\Delta \epsilon_{0} \neq 0$ the value of $T_{n}^{(+)}$is larger. This means that $\ln \left(T / T_{n}^{(+)}\right)$is smaller than in the preceeding case at the same $T$. Second, the $\Delta \epsilon_{0}$ term can decrease the contribution to the denominator and thus further suppresses a high $-T$ decrease of $\Delta_{\rho}$.

There is yet a third case which may be applicable to the atoms on either end of the family. This is the case in which $\Delta \epsilon_{0}$ is so large that the corresponding $T_{n}^{(+)}$is comparable to or larger than the temperature $T$ at which we are comparing the resistivities. In this case the shift $\Lambda$ will not have changed much $\left(T \leqslant T_{n}^{(+)}\right)$and would not vary like $\log (T)$, and the resistivity will have undergone little or no change.

This qualitative discussion thus indicates that the behavior of the resistivity across the iron group at both low and high temperatures can be rationalized by the effects presented in this paper.

Further examination of other consequences of the theoretical ideas presented here are being pursued and will be discussed elsewhere.

\section{SUMMARY}

The logical consequences of considering the ionic approach of Schrieffer and Hirst in a thermodynamic quantum-mechanical formalism which includes impurity lifetime broadening has been presented in some detail. Up to a question of overall temperature scale, or equivalently, the question of the lifetime broadening of the relevant many electron ICEE impurity resonances, the consequences of this ionic perspective seem to describe impurity systems remarkably like the physical alloys. The different types of temperature dependences of bulk properties and related effects seem very much like the experimental behavior of many alloy systems. Furthermore, our calculations show a possible intimate link between Kondolike behavior of transport properties, Pauli-like behavior of the paramagnetic susceptibility, and valence fluctuations of the transition metal or actinide series atoms.

\section{ACKNOWLEDGMENTS}

The author gratefully acknowledges the helpful conversations of a large number of colleagues during the long study of this problem: R. Silsbee, A. L. Ritter, J. Gardner, C. D. Williams, J. Long, and $R$. Orbach, to name a few. Their good physical intuition and sound physical understanding has been incorporated where ever possible. Any errors are the sole responsibility of the author. 


\section{APPENDIX A: DETAILS OF THE SECULAR MATRIX}

As indicated in Eq. (4.7), the first application of the Gram-Schmidt orthogonalization procedure yields a symmetrized form of the secular equation, but one which contains vectors $c_{d \bar{s}}^{\dagger} c_{\mathrm{k}} \bar{s} c_{d s}$ and $c_{\mathrm{k}} \bar{s} c_{d s} c_{d s}$, which are not orthogonal to $\phi_{d s}^{(r)}$. To carry out the orthogonalization it is necessary to find the overlap between these vectors. This is the same as calculating the matrix element

$$
\left(\left(\phi_{d s}^{(r)}, L \phi_{d s}^{(r)}\right)\right)=E_{s}^{(r)}+\Delta E_{s}^{(r)},
$$

where

$$
\Delta E_{s}^{(r)}=r \sum_{\overrightarrow{\mathrm{k}}} \frac{V_{\overrightarrow{\mathrm{k}} d}}{\left\langle N_{d \bar{s}}^{(r)}\right\rangle}\left(\left(\phi_{d s}^{(r)},\left(c_{d \bar{s}}^{+} c_{\mathrm{k} \bar{s}}-c_{\mathrm{k}}^{\dagger} \bar{s} c_{d \bar{s}}\right) c_{d s}\right)\right) .
$$

The evaluation of the commutators in (A2) yields

$$
\begin{array}{r}
\left\langle N_{d \bar{s}}^{(r)}\right\rangle \Delta E_{s}^{(r)}=\sum_{\overrightarrow{\mathrm{k}}} V_{\overrightarrow{\mathrm{k}} d}\left\langle\left\langle N_{d s}^{(+)}\right\rangle^{1 / 2}\left\langle\phi_{d s}^{+(+)} c_{\overrightarrow{\mathrm{k}} \bar{s}}\right\rangle\right. \\
\left.-\left\langle N_{d s}^{(-)}\right\rangle^{1 / 2}\left\langle c_{\overrightarrow{\mathrm{k}} \bar{s}}^{\dagger} \phi_{d s}^{(-)}\right\rangle\right),
\end{array}
$$

or, as written in Eq. (4.10) in terms of the $\lambda_{s}^{(r)}$ parameter,

$$
\Delta E_{s}^{(r)}=-\frac{\Delta}{\pi} \frac{\left\langle n_{d s}\right\rangle}{\left\langle N_{d s}^{(r)}\right\rangle}\left(\lambda_{s}^{(+)}-\frac{1-\left\langle n_{d s}\right\rangle}{\left\langle n_{d s}\right\rangle} \lambda_{s}^{(-)}\right) .
$$

To carry out the Schmidt orthogonalization we must add in and subtract out the parts of the vectors $c_{d \bar{s}}^{\dagger} c_{\bar{k}} \bar{s} c_{d s}$ and $c_{\bar{k}}^{\dagger} \bar{s} c_{d \bar{s}} c_{d s}$ which are along $\phi_{d s}^{(r)}$.

The resulting row of the secular equation becomes

$$
\begin{aligned}
L \phi_{d s}^{(r)}= & \left(E_{s}^{(r)}-\frac{\Delta}{\pi} \frac{\left\langle n_{d s}\right\rangle}{\left\langle N_{d \vec{s}}^{(r)}\right\rangle^{(r)}} \Lambda_{s}\right) \phi_{d s}^{(r)}+\left\langle N_{d \bar{s}}^{(r)}\right\rangle^{1 / 2} \sum_{\overrightarrow{\mathrm{k}}} V_{\overrightarrow{\mathrm{k}} d} c_{\overrightarrow{\mathrm{k}} s} \\
& +r\left\langle N_{d \vec{s}}^{(r)}\right\rangle^{1 / 2} \sum_{\overrightarrow{\mathrm{k}}} V_{\overrightarrow{\mathrm{k}} d} \phi_{d \overrightarrow{\mathrm{k}} s} \\
& +\frac{r}{\left\langle N_{d \bar{s}}^{(r)}\right\rangle^{1 / 2}} \sum_{\overrightarrow{\mathrm{k}}} V_{\overrightarrow{\mathrm{k}} d}\left\|\tilde{\phi}_{\overrightarrow{d \overrightarrow{\mathrm{k}}} d s}^{(r)}\right\| \phi_{\overrightarrow{d \overrightarrow{\mathrm{k}}} d s}^{(r)} \\
& -\frac{r}{\left\langle N_{d \bar{s}}^{(r)}\right\rangle^{1 / 2}} \sum_{\overrightarrow{\mathrm{k}}} V_{\overrightarrow{\mathrm{k}} d}\left\|\tilde{\phi}_{\overrightarrow{\mathrm{k}} d d s}^{(r)}\right\| \phi_{\overrightarrow{\mathrm{k}} d d s}^{(r)}
\end{aligned}
$$

Here the vectors $\phi_{d \mathrm{k} d s}^{(r)}$ and $\phi_{\mathrm{k}}^{(r)} d d s$ are the unit vectors for the vectors

$$
\begin{aligned}
& \tilde{\phi}_{d \mathrm{k} d s}^{(r)}=c_{d s}^{\dagger} c_{\overline{\mathrm{k}}} \bar{s} c_{d s}-K_{\overrightarrow{\mathrm{k}} d s}^{(r)} \phi_{d s}^{(r)}, \\
& \tilde{\phi}_{\overrightarrow{\mathrm{k}} d d s}=c_{\mathrm{k} \bar{s}}^{\dagger} c_{d s} c_{d s}-K_{\mathrm{k} d s}^{(r)} \phi_{d s}^{(r)},
\end{aligned}
$$

where

$$
\begin{aligned}
K_{\mathrm{k} d s}^{(r)}=\frac{r}{\left\langle N_{d \bar{s}}^{(r)}\right\rangle^{1 / 2}} & \left\langle\langle N _ { d s } ^ { ( + ) } \rangle ^ { 1 / 2 } \left\langle\phi_{d \bar{s}}^{\dagger(+)} c_{\overrightarrow{\mathrm{k}} \bar{s}}^{(+)} .\right.\right. \\
& \left.-\left\langle N_{d s}^{(-)}\right\rangle^{1 / 2}\left\langle c_{\mathrm{k} \bar{s}}^{+} \phi_{d \bar{s}}^{(-)}\right\rangle\right) .
\end{aligned}
$$

The quantities $\left\|\tilde{\phi}_{d \overrightarrow{\mathrm{k}} d s}^{(r)}\right\|$ and $\left\|\tilde{\phi}_{\mathrm{k} d d s}^{(r)}\right\|$ are the norms of the corresponding vectors.

At this stage, we start to utilize the assumed weakness of the coupling between the conduction electrons and the impurity resonances. In the evaluation of the self-energy of the impurity resonances, the weak-coupling condition will be invoked, and the only term for the self-energy which will be evaluated is (written in notation of Sec. II)

$$
e_{n}=E_{n}+\sum_{l(\neq n)} \frac{\left|V_{l n}\right|^{2}}{\omega-e_{l}(n, \omega)} .
$$

The argument justifying this expression is that the third-and higher-order terms will be smaller and will be neglected. In what follows we make the further approximation of determining each matrix element $V_{l n}$ to only second order in $V_{\mathbb{k}_{d}}$, or equivalently to first order in $\Delta=\pi N(0) V_{\vec{k} d}^{2}$.

In most circumstances, a determination to lowest order in $\Delta$ would also imply that our determination of $e_{l}(n, \omega)$ should be only to zeroth order in $\Delta$. For a low-density system, this would be correct, but with a degenerate Fermi system the sum over intermediate states can lead in the thermodynamic limit to essential singularities of the self-energy for some values of the coupling constant. This is a general feature of secular equations where the eigenvalues are not necessarily entire functions of the matrix elements. As we proceed the reduced self-energies and all other energies will be evaluated to whatever lowest order in $\Delta$ which is required to yield nonsingular results.

As a first step toward evaluating the self-energy, we must list the states " $l$ " which have to be included. As can be seen in the relevant row of the secular equation these vectors include

$$
c_{\overrightarrow{\mathrm{k}} s}, \phi_{d \overrightarrow{\mathrm{k}} s}, \phi_{d \overrightarrow{\mathrm{k}} d s}, \phi_{\overrightarrow{\mathbf{k}} d d s} .
$$

The matrix elements required are

$$
\begin{aligned}
& \left(\left(c_{\overrightarrow{\mathrm{k}} s}, L \phi_{d s}^{(r)}\right)\right)=V_{\overrightarrow{\mathrm{k}} d}\left\langle N_{d \vec{s}}^{(r)}\right\rangle^{1 / 2}, \\
& \left(\left(\phi_{d \overrightarrow{\mathrm{k}} s}, L \phi_{d s}^{(r)}\right)\right)=r V_{\overrightarrow{\mathbf{k}} d}\left\langle N_{d \vec{s}}^{(-r)}\right\rangle^{1 / 2}, \\
& \left(\left(\phi_{d \overrightarrow{\mathrm{k}} d s}, L \phi_{d s}^{(r)}\right)\right)=r V_{\overrightarrow{\mathbf{k}} d}\left\|\tilde{\phi}_{d \overrightarrow{\mathrm{k}} d s}\right\| /\left\langle N_{d \vec{s}}^{(r)}\right\rangle^{1 / 2}, \\
& \left(\left(\phi_{\overrightarrow{\mathrm{k}} d d s}, L \phi_{d s}^{(r)}\right)\right)=-r V_{\overrightarrow{\mathrm{k}} d}\left\|\tilde{\phi}_{\overrightarrow{\mathrm{k}} d d s}\right\| /\left\langle N_{d \vec{s}}^{(r)}\right\rangle^{1 / 2} .
\end{aligned}
$$

We next evaluate the matrix elements to lowest relevant order in $V_{\vec{k} d}$. For (A13) and (A14) this means

$$
\left\|\tilde{\phi}_{d \overrightarrow{\mathrm{k}} d s}\right\|=\left\|c_{d \bar{s}}^{\dagger} c_{\overrightarrow{\mathrm{k}} \vec{s}} c_{d s}\right\|+O\left(V_{\overrightarrow{\mathrm{k}} d}\right)
$$

and

$$
\left\|\tilde{\phi}_{\overrightarrow{\mathbf{k}} d d s}\right\|=\left\|c_{\overrightarrow{\mathbf{k}} \vec{s}}^{\dagger} c_{d \vec{s}} c_{d s}\right\|+O\left(V_{\overrightarrow{\mathbf{k}} d}\right) .
$$

It is straightforward to verify that

$$
\left\|c_{d \bar{s}}^{\dagger} c_{\vec{k} \vec{s}} c_{d s}\right\|^{2}=\left(1-\left\langle n_{d s}\right\rangle\right) f_{e}^{(-)}+\left\langle\left(n_{d s}-n_{d \bar{s}}\right) n_{\overrightarrow{\mathrm{k}} \bar{s}}\right\rangle
$$


and

$\left\|c_{\overrightarrow{\mathrm{k}} \bar{s}}^{\dagger} c_{d \bar{s}} c_{d s}\right\|^{2}=\left\langle n_{d s}\right\rangle f_{s}^{(+'}+\left\langle n_{\overrightarrow{\mathrm{k}} \bar{s}}\left(1-n_{d s}-n_{d \bar{s}}\right)\right\rangle$,

where, as before,

$$
f_{s}^{(r)}=\left\langle\phi_{d s}^{\dagger(r)} \phi_{d s}^{(r)}\right\rangle \text {. }
$$

Now the norms involve a knowledge of expectations of the sort $\left\langle n_{\overrightarrow{\mathbf{z}} s_{1}} n_{d s_{2}}\right\rangle$. Such quantities can be evaluated from two-particle correlation functions, but at this point we adopt the form for such coefficients which holds to zeroth order in $V_{\overrightarrow{\mathbb{k}} d}$. This approximation does not represent the neglect of any singular terms and so should be accurate in the weak coupling limit. Thus,

$$
\left\langle n_{d s_{1}} n_{\overrightarrow{\mathbf{k}} s_{2}}\right\rangle=\left\langle n_{d s_{1}}\right\rangle\left\langle n_{\overrightarrow{\mathbf{k}} s_{2}}\right\rangle+O\left(V_{\vec{k} \mathfrak{k} d}\right) .
$$

These approximations have the obvious advantage that our self-consistency equations are closed, in that all thermal expectations can be computed by a limited number of correlation functions. The different contributions to the self-energy can now be written out:

$$
\begin{aligned}
\Sigma\left(\phi_{d s}^{(r)} ; \omega\right)= & \left\langle N_{d}^{\left(\frac{r}{s}\right)}\right\rangle \sum_{\overrightarrow{\mathrm{k}}} \frac{V_{\overrightarrow{\mathrm{k} d}}^{2}}{\omega-\epsilon_{\overrightarrow{\mathrm{k}} s}} \\
& +\left\langle N_{d \bar{s}}^{(-r)}\right\rangle \sum_{\overrightarrow{\mathrm{k}}} \frac{V_{\overrightarrow{\mathrm{k}} d}^{2}}{\omega-e\left(\phi_{\overrightarrow{\mathrm{k}} d s} ; \omega\right)}+\frac{1}{\left\langle N_{d \bar{s}}^{(\underline{r})}\right\rangle} Y_{s}(\omega),
\end{aligned}
$$

where

$$
\begin{aligned}
Y_{s}(\omega)=\sum_{\overrightarrow{\mathrm{k}}}\left\{V _ { \vec { \mathrm { k } } d } ^ { 2 } \left[\left(1-\left\langle n_{d s}\right\rangle\right) f_{s}^{(-)}+\left(\left\langle n_{d s}\right\rangle\right.\right.\right. \\
\left.\left.\left.-\left\langle n_{d \vec{s}}\right\rangle\right)\left\langle n_{\overrightarrow{\mathrm{k}} \vec{s}}\right\rangle\right] /\left[\omega-e\left(\phi_{d \overrightarrow{\mathrm{k}} s} ; \omega\right)\right]\right\} \\
+\sum_{\overrightarrow{\mathrm{k}}}\left\{V _ { \vec { \mathbb { k } } d } ^ { 2 } \left[\left\langle n_{d s}\right\rangle f_{s}^{(+)}+\left(1-\left\langle n_{d s}\right\rangle\right.\right.\right. \\
\left.\left.\left.-\left\langle n_{d \vec{s}}\right\rangle\right)\left\langle n_{\overrightarrow{\mathrm{k}} \vec{s}}\right\rangle\right] /\left[\omega-e\left(\phi_{\overrightarrow{\mathrm{k}} d d s} ; \omega\right)\right]\right\}
\end{aligned}
$$

To carry out the approximation scheme more carefully we must now determine good approximations for the various reduced energy denominators $e(\phi ; \omega)$. These energies will contain a diagonal energy and a reduced self-energy arising from the off-diagonal matrix elements. In all cases the diagonal energy must be determined, but one might hope to approximate the reduced self-energy because of our weak-coupling limitation. Care must be taken in these approximations to examine the effect of sums over intermediate states. In the case where the matrix elements are independent of $n$, we may sum over intermediate states in a convergent power series in the weak coupling. Thus, for example, we approximate

$$
\sum_{\overrightarrow{\mathrm{z}}} \frac{V_{\mathrm{k} d}^{2}}{\omega-e\left(\phi_{\overrightarrow{\mathrm{k}} d s} ; \omega\right)} \approx \sum_{\overrightarrow{\mathrm{k}}} \frac{V_{\overrightarrow{\mathrm{k}} d}^{2}}{\omega-\epsilon_{\overrightarrow{\mathrm{k}} s}} \equiv \gamma(\omega) .
$$

Similarly, for those terms which involve the same class of integrals, we write the same approximation. This yields for $Y_{s}$ the following result

$$
\begin{aligned}
Y_{s}(\omega)= & \left(1-\left\langle n_{d s}\right\rangle\right) f_{\vec{s}}^{(-)} \gamma(\omega)+\left\langle n_{d s}\right\rangle f_{\vec{s}}^{(+)} \gamma\left(\omega-2 \epsilon_{d}-U\right) \\
& +\left(\left\langle n_{d s}\right\rangle-\left\langle n_{d \vec{s}}\right\rangle\right) \sum_{\overrightarrow{\mathbf{k}}} \frac{V_{\overrightarrow{\mathrm{k} d} d}^{2}\left\langle n_{\overrightarrow{\mathrm{k}} \vec{s}}\right\rangle}{\omega-e\left(\phi_{d \overrightarrow{\mathrm{k} d} d s} ; \omega\right)} \\
& +\left(1-\left\langle n_{d s}\right\rangle-\left\langle n_{d \vec{s}}\right\rangle\right) \sum_{\overrightarrow{\mathbf{k}}} \frac{V_{\mathrm{k} d}^{2}\left\langle n_{\overrightarrow{\mathbf{k}} \vec{s}}\right\rangle}{\omega-e\left(\phi_{\overrightarrow{\mathbf{k}} d d s} ; \omega\right)} .
\end{aligned}
$$

Now for the dilute alloy, it is entirely appropriate to approximate the $\left\langle n_{\overrightarrow{\mathrm{k} s}}\right\rangle$ factor by the Fermi function

$$
\left\langle n_{\overrightarrow{\mathrm{k}} \overrightarrow{\mathrm{s}}}\right\rangle=\left[\exp \left(\beta \epsilon_{\overrightarrow{\mathrm{k}} \overrightarrow{\mathrm{s}}}\right)+1\right]^{-1} .
$$

Having done this it is now necessary to be careful about how the denominators $e(\phi ; \omega)$ are approximated. In particular, it is not admissable to neglect the imaginary part of the reduced self-energy, because one of the contributions to these integrals is of the form $\Delta \ln \Delta$, where $\Delta$ $=\pi\left(V_{\mathbb{E} d}^{2}\right) N(0)$, and this does not have a small $-\Delta$ power series expansion about $\Delta=0$. This means that the lowest-order contribution from these Fermi function integrals must include a lifetime broadening as well as any shifts. Thus the evaluation of these terms requires a specification of both the real and imaginary parts, $e_{1}$ and $e_{2}$, of $e$. In order to determine these it is straightfor ward to observe that

$$
L c_{d \bar{s}-\overline{\mathrm{k}} \vec{s}}^{\dagger} c_{d s}=\epsilon_{\overrightarrow{\mathrm{k}} \vec{s}} c_{d \vec{s}}^{\dagger} c_{\overrightarrow{\mathrm{k}} \vec{s}} c_{d s}+O\left(V_{\overrightarrow{\mathrm{k}} d}\right)
$$

and

$$
L c_{\overrightarrow{\mathrm{k}} \vec{s}}^{\dagger} c_{d \vec{s}} c_{d s}=\left(2 \epsilon_{d}+U-\epsilon_{\overrightarrow{\mathrm{k}} \vec{s}}\right) c_{\overrightarrow{\mathrm{k}} \vec{s}}^{\dagger} c_{d \vec{s}} c_{d s}+O\left(V_{\overrightarrow{\mathrm{k}} d}\right) .
$$

Note that these energies involve just the energy of a conduction electron or hole along with the twoelectron or non-electron impurity state.

To proceed in greater detail with the reduced self-energy of each of these intermediate states is to introduce quite a bit more detail into this problem than is useful. Therefore we set

$$
e_{2}(\phi ; \omega)=\Delta^{\prime}=t^{\prime} \Delta
$$

where $t^{\prime}$ is a dimensionless parameter (for the most part $\left.t^{\prime} \approx 1\right)$. Using the results of Appendix $C$, we have immediately,

$$
\sum_{\overrightarrow{\mathrm{k}}} \frac{V_{\overrightarrow{\mathrm{k}} d}^{2}\left\langle n_{\overrightarrow{\mathrm{r}} \vec{s}}\right\rangle}{\omega-\epsilon_{\overrightarrow{\mathrm{k}} s}-i \Delta^{\prime}}=\frac{\Delta}{\pi} \boldsymbol{s}_{D}\left(\omega, \Delta^{\prime}\right),
$$

and can rewrite $Y_{s}(\omega)$ as follows: 


$$
\begin{aligned}
Y_{s}(\omega)= & \left(1-\left\langle n_{d s}\right\rangle\right) f_{\bar{s}}^{(-)} \gamma(\omega)+\left\langle n_{d s}\right\rangle f_{\bar{s}}^{(+)} \gamma\left(\omega-2 \epsilon_{d}-U\right) \\
& +\frac{\Delta}{\pi}\left(\left\langle n_{d s}\right\rangle-\left\langle n_{d \vec{s}}\right\rangle\right) \boldsymbol{s}_{D}\left(\omega, \Delta^{\prime}\right) \\
& +\frac{\Delta}{\pi}\left(1-\left\langle n_{d s}\right\rangle-\left\langle n_{d \vec{s}}\right\rangle\right) \\
& \times \int_{-D}^{D} \frac{f_{0}(\epsilon) d \epsilon}{\omega-2 \epsilon_{d}-U-i \Delta^{\prime}-\epsilon} .
\end{aligned}
$$

The integrals defining $\boldsymbol{S}_{D}$, and in Appendix B the integrals defining $\lambda_{s}^{(r)}$, are quite simple to or$\operatorname{der} 1 / D$ if the impurity resonances can be regarded as Lorentzians with constant lifetime broadening and energy-independent shift. In the following we seek two small parameters $\delta E_{s}^{(r)}$ and $\Gamma_{s}^{(r)}$, so that

$$
e_{s}^{(r)}\left(E_{s}^{(r)}+S E_{s}^{(r)}\right)=E_{s}^{(r)}+\delta E_{s}^{(r)}+i \Gamma_{s}^{(r)}
$$

In terms of these parameters each resonance of the impurity will be a simple Lorentzian

$$
\left(\left(\phi_{d s}^{(r)},(\omega-L)^{-1} \phi_{d s}^{(r)}\right)\right)=\left(\omega-E_{s}^{(r)}-\delta E_{s}^{(r)}-i \Gamma_{s}^{(r)}\right)^{-1} .
$$

It is shown in Appendix B that if Eq. (A29) gives the form of the resonance, then to order $1 / D$ the value of $\lambda_{s}^{(r)}$ is given by

$$
\lambda_{s}^{(r)}=g_{D}^{\prime}\left(E_{\frac{(}{s}}^{(r)}+\delta E_{s}^{(r)}, \Gamma_{s}^{(r)}\right),
$$

where $\boldsymbol{S}_{D}^{\prime}$ is the real part of $\boldsymbol{g}_{D}$ as defined in Appendix $C$. Note the peculiarity of the notation that $\lambda_{s}^{(r)}$ depends on the $\bar{s}$ resonance energies. The imaginary part of $g_{D}$ is defined in (C13) in terms of $\hat{f}(E, \Gamma)$, and it can be easily shown that

$$
f_{s}^{(r)}=\hat{f}\left(E_{s}^{(r)}+\delta E_{s}^{(r)}, \Gamma_{s}^{(r)}\right) .
$$

All that remains is to derive the functional form for $\delta E_{s}^{(r)}$ and $\Gamma_{s}^{(r)}$. The calculation for $\delta E_{s}^{(r)}$ involves evaluating $Y_{s}\left(E_{s}^{(r)}+\delta E_{s}^{(r)}\right)$ and substituting this into (A25). After some algebra the expression for $\delta E_{s}^{(+)}$including corrections to order $\Delta$ is easily found:

$$
\begin{aligned}
\delta E_{s}^{(+)}= & -\frac{\Delta}{\pi}\left(\lambda_{s}^{(+)}-\lambda_{s}^{(-)}\right)+\frac{\Delta}{\pi}\left(\left\langle n_{d s}\right\rangle-\left\langle n_{d-\bar{s}}\right\rangle\right)\left[\frac{\partial \lambda_{s}^{(+)}}{\partial E_{s}^{(+)}}\left(\delta E_{s}^{(+)}-\delta E_{\bar{s}}^{(+)}\right)+\frac{\partial \lambda_{s}^{(+)}}{\partial \Gamma_{\bar{s}}^{(+)}}\left(\Delta^{\prime}-\Gamma_{\frac{s}{s}}^{(+)}\right)\right] \\
& +\frac{\Delta}{\pi}\left(1-\left\langle n_{d s}\right\rangle-\left\langle n_{d-\bar{s}}\right\rangle\right)\left[-\frac{\partial \lambda_{s}^{(-)}}{\partial E_{\bar{s}}^{(-)}}\left(\delta E_{s}^{(+)}+\delta E_{s}^{(-)}\right)+\frac{\partial \lambda_{s}^{(-)}}{\partial \Gamma_{s}^{(-)}}\left(\Delta^{\prime}-\Gamma_{\bar{s}}^{(-)}\right)\right] .
\end{aligned}
$$

Similar equations are found for $\delta E_{s}^{(-)}$which differ only in the second and third lines. The correction coefficients in (A32) vanish exactly in the absence of a magnetic field and if the impurity is on the average neutral. In any case, the correction terms are quite small and can be neglected. This gives the result in Eq. (4.24):

$$
\delta E_{s}^{(r)}=-\frac{\Delta}{\pi}\left(\lambda_{s}^{(+)}-\lambda_{s}^{(-)}\right)
$$

Similar calculations yield the following lowestorder expressions:

$$
\Gamma_{s}^{(+)}=\Delta\left[1+f_{s}^{(+)}+\frac{2-\left\langle n_{d s}\right\rangle}{\left\langle n_{d s}\right\rangle} f_{s}^{(-)}\right]
$$

and

$$
\Gamma_{s}^{(-)}=\Delta\left(1+f_{s}^{(+)}+f_{s}^{(-)}\right)
$$

These last equations have also neglected correction terms like those in (A32), which terms are of order $\Delta^{2}$ and will not be discussed here.

\section{APPENDIX B: DERIVATION OF THE $\lambda_{s}^{(r)}$}

In this Appendix, the expression for $\lambda_{s}^{(r)}$ given by Eq. (4.22) is derived within the resonance approximation of (A29). We want to evaluate

$$
\sum_{\overrightarrow{\mathbf{k}}} V_{\overrightarrow{\mathbf{k}} d}\left\langle c_{\overrightarrow{\mathbf{k}} \vec{s}}^{\dagger} \phi_{d \vec{s}}^{(\underline{r})}\right\rangle=\mathcal{F}_{\omega}\left[\sum_{\overrightarrow{\mathbf{k}}} V_{\overrightarrow{\mathbf{k}} d}\left(\left(c_{\overrightarrow{\mathbf{k}} \vec{s}},(\omega-L)^{-1} \phi_{d}^{\left(\frac{r}{s}\right)}\right)\right)\right],
$$

where $\mathcal{F}_{\omega}[\cdots]$ is the Hamann-Bloomfield functional

$$
\begin{aligned}
\mathcal{F}_{\omega}\left[\left(\left(A,(\omega-L)^{-1} B\right)\right)\right]=\int_{-\infty}^{\infty} & \frac{d \omega}{\pi} f_{0}(\omega) \\
& \times \operatorname{Im}\left(\left(A,(\omega-i \delta-L)^{-1} B\right)\right) .
\end{aligned}
$$

Using the Lorentizian form for the diagonal matrix elements of the secular equation as determined in Appendix A, we have

$\operatorname{Im}\left(\left(c_{\overrightarrow{\mathrm{k}} \vec{s}},(\omega-i \delta-L)^{-1} \phi_{d \bar{s}}^{(r)}\right)\right)=V_{\overrightarrow{\mathrm{k}} d}\left\langle N_{d \bar{s}}^{(r)}\right\rangle^{1 / 2}\left[\frac{\omega-\epsilon_{\overrightarrow{\mathrm{k}} \bar{s}}}{\left(\omega-\epsilon_{\overrightarrow{\mathrm{k}} \vec{s}}\right)^{2}+\delta^{2}} \frac{\Gamma_{\vec{s}}^{(r)}}{\left(\omega-E_{\vec{s}}^{(r)}-\delta E_{\bar{s}}^{(r)}\right)^{2}+\left(\Gamma_{\vec{s}}^{(r)}\right)^{2}}+\frac{\pi \delta\left(\omega-\epsilon_{\overrightarrow{\mathrm{k}} \vec{s}}\right)\left(\omega-E_{s}^{(r)}-\delta E_{s}^{(r)}\right)}{\left(\omega-E_{\vec{s}}^{(r)}-\delta E_{\bar{s}}^{(r)}\right)^{2}+\left(\Gamma_{\vec{s}}^{(r)}\right)^{2}}\right]$. 
Evaluating the functional $\mathcal{F}_{\omega}$ we have

$$
\left\langle c_{\overrightarrow{\mathbf{k}} \bar{s}}^{t} \phi_{d \bar{s}}^{(r)}\right\rangle=V_{\overrightarrow{\mathbf{k}} d}\left\langle N_{d \vec{s}}^{(r)}\right\rangle^{1 / 2}\left[\boldsymbol{g}_{1}\left(\epsilon_{\overrightarrow{\mathrm{k}}-\bar{s}}\right)+g_{2}\left(\epsilon_{\overrightarrow{\mathbf{k}} \vec{s}}\right)\right],
$$

where

$$
\begin{aligned}
g_{1}(\epsilon)= & \int_{-\infty}^{\infty} \frac{d \omega}{\pi} \frac{f_{0}(\omega)(\omega-\epsilon)}{(\omega-\epsilon)^{2}+\delta^{2}} \\
& \times \frac{\Gamma_{s}^{(r)}}{\left(\omega-E_{\frac{s}{s}}^{(r)}-\delta E_{\frac{(r)}{s}}^{(2)}+\left(\Gamma_{\frac{s}{s}}^{(r)}\right)^{2}\right.}, \\
g_{2}(\epsilon)= & \frac{f_{0}(\epsilon)\left(\epsilon-E_{\frac{s}{s}}^{(r)}-\delta E_{\frac{s}{s}}^{(r)}\right)}{\left(\epsilon-E_{\frac{(r)}{s}}^{\left(\frac{r}{s}\right.}-\delta E_{\frac{(r)}{s}}^{(r)}+\left(\Gamma_{\frac{(r)}{s}}^{2}\right)^{2}\right.} .
\end{aligned}
$$

The evaluation of the sum over $\vec{k}$ yields

$$
\begin{aligned}
& \sum_{\overrightarrow{\mathbf{k}}} V_{\overrightarrow{\mathbf{k}} d}\left\langle c_{\overrightarrow{\mathbf{k}} \bar{s}}^{\dagger} \phi_{d \bar{s}}^{(r)}\right\rangle \approx V_{\vec{k} d}^{2}\left\langle N_{d \bar{s}}^{(r)}\right\rangle^{1 / 2}\left[\sum_{\overrightarrow{\mathbf{k}}}\left[\mathscr{g}_{1}\left(\epsilon_{\overrightarrow{\mathbf{k}} s}\right)+\mathscr{g}_{2}\left(\epsilon_{\overrightarrow{\mathbf{k}} s}\right)\right]\right] \\
& \approx \frac{\Delta}{\pi}\left\langle N_{d s}^{(\underline{x})}\right\rangle^{1 / 2}\left[\frac{1}{N(0)} \sum_{\overrightarrow{\mathbf{k}}} \mathfrak{g}_{2}\left(\epsilon_{\overrightarrow{\mathbf{k}} s}\right)\right. \\
& \left.+\frac{1}{N(0)} \sum_{\overrightarrow{\mathrm{k}}} g_{1}\left(\epsilon_{\overrightarrow{\mathrm{r}} s}\right)\right] \text {, }
\end{aligned}
$$

where $N(0)$ is the density of states at the Fermi surface. Comparing the definitions of $\boldsymbol{g}_{D}^{\prime}(E, \Gamma)$ (Appendix C) and $\lambda_{s}^{(r)}$ we see that

$$
\lambda_{s}^{(r)}=g_{D}^{\prime}\left(E_{s}^{(r)}+\delta E_{\frac{s}{s}}^{(r)}, \Gamma_{\frac{s}{(r)}}^{(r)}+\delta \lambda_{s}^{(r)} .\right.
$$

In the following we show that $\delta \lambda_{s}^{(r)}$ is of order $1 / D$ and thus can be neglected when the cutoff becomes large. In order to estimate this integral it becomes necessary to use a more realistic density of states for the conduction electrons, which still cuts off at energies of the order of $D$. For this purpose, we adopt the Lorentzian density of states of Hamann and Bloomfield

$$
N(\epsilon)=\frac{2 N(0) D^{2}}{\pi\left(\epsilon^{2}+D^{2}\right)}
$$

Actually, all calculations of this model could have been carried out using the density of states (B9), but it then would have been somewhat more complicated. The integral to be done is

$\delta \lambda_{s}^{(r)}=\int_{-\infty}^{+\infty} d \epsilon \frac{N(\epsilon)}{N(0)} \int_{-\infty}^{\infty} \frac{d \omega}{\pi} \frac{f_{0}(\omega)(\omega-\epsilon)}{(\omega-\epsilon)^{2}+\delta^{2}} \frac{e_{2}}{\left(\omega-e_{1}\right)^{2}+e_{2}^{2}}$

The evaluation of the required contour integrations is straightforward, but lengthy, and will not be reproduced here. The essential point is that integrating first over $\epsilon$ yields an integral over $\omega$ of the form

$$
\int_{-\infty}^{\infty} \frac{d \omega}{\pi} \frac{2 D \omega}{\omega^{2}+D^{2}} \frac{f_{0}(\omega) e_{2}}{\left(\omega-e_{1}\right)^{2}+e_{2}^{2}},
$$

which is clearly of order $1 / D$ as $D \rightarrow \infty$ and can be neglected in comparison with the $\mathfrak{s}_{D}^{\prime}$ term.

\section{APPENDIX C: PROPERTIES OF $g_{D}(E, \Gamma)$}

For the parameter ranges of this work we want $\delta_{D}$ for large $D$, and we will only list expressions for that limit. The definition is

$$
\boldsymbol{s}_{D}(E, \Gamma)=\int_{-D}^{D} d \epsilon \frac{f_{0}(\epsilon)}{E-i \Gamma-\epsilon},
$$

where

$$
f_{0}(\epsilon)=\left(e^{\beta \epsilon}+1\right)^{-1}
$$

This integral may be written as

$$
\delta_{D}(E, \Gamma)=I_{1}+I_{0} \text {, }
$$

where

$$
\begin{aligned}
& I_{0}=\ln (D+E-i \Gamma)-\ln (E-i \Gamma), \\
& I_{1}=-\int_{0}^{\infty} \frac{d \epsilon}{e^{\beta \epsilon}+1} \frac{2 \epsilon}{\epsilon^{2}+\gamma^{2}},
\end{aligned}
$$

and

$$
\gamma=\Gamma+i E
$$

In the derivation of $I_{1}$ the integration range has been extended from $D$ to infinity. This will give errors only of order $e^{-\beta D}$. The integral $I_{1}$ may be written in terms of digamma functions if the following identity is used:

$$
\left(e^{\beta \epsilon}+1\right)^{-1}=\left(e^{\beta \epsilon}-1\right)^{-1}-2\left(e^{2 \beta \epsilon}-1\right)^{-1} .
$$

Substituting (C6) into (C4) and using the definition of the digamma function, ${ }^{88}$

$$
\int_{0}^{\infty} \frac{d x}{e^{x}-1} \frac{x}{x^{2}+\gamma^{2}}=\frac{1}{2}\left[\ln \left(\frac{\gamma}{2 \pi}\right)-\frac{\pi}{\gamma}-\psi\left(\frac{\gamma}{2 \pi}\right)\right],
$$

yields $I_{1}$ in terms of the variable $z=\beta \gamma / 2 \pi$ as

$$
I_{1}(z)=\ln (4 z)+\psi(z)-2 \psi(2 z),
$$

where $\psi(z)$ is the digamma function. Using the duplication formula for the digamma function ${ }^{89}$ yields another simpler form

$$
I_{1}(z)=\ln (z)-\psi\left(\frac{1}{2}+z\right) \text {. }
$$

This last form yields for $\boldsymbol{S}_{D}(E, \Gamma)$ the following expression,

$$
\boldsymbol{S}_{D}(E, \Gamma)=\ln \left[\frac{\beta}{2 \pi}(D+E-i \Gamma)\right]+i \frac{1}{2} \pi-\psi\left(\frac{1}{2}+z\right),
$$

which is accurate to order $e^{-\beta D}$.

The temperature behavior of $\boldsymbol{s}_{D}(E, \Gamma)$ is determined by two characteristic temperatures $T_{D}$ and $T_{\Gamma, E}$, where

$$
k_{B} T_{D}=\frac{1}{\pi}\left[(D+E)^{2}+\Gamma^{2}\right]^{1 / 2},
$$




$$
k_{B} T_{\Gamma, E}=\frac{1}{\pi}\left(E^{2}+\Gamma^{2}\right)^{1 / 2} .
$$

For use in other appendices, define the real and imaginary part of $S_{D}$ in the following way:

$$
\boldsymbol{s}_{D}(E, \Gamma)=\boldsymbol{g}_{D}^{\prime}(E, \Gamma)+i \pi \hat{f}(E, \Gamma) \text {. }
$$

Then it is easy to show that if $T<\frac{1}{2} T_{\mathrm{r}, E}$, then

$$
\begin{aligned}
& \boldsymbol{\delta}_{D}^{\prime}(E, \Gamma) \approx \ln \left(\frac{T_{D}}{T_{\Gamma, E}}\right)-\left(k_{B} T\right)^{2} \frac{1}{6} \pi^{2} \frac{\left(\Gamma^{2}-E^{2}\right)}{\left(\Gamma^{2}+E^{2}\right)^{2}}, \\
& \hat{f}(E, \Gamma) \approx \frac{1}{2}-\frac{1}{\pi} \tan ^{-1}\left(\frac{E}{\Gamma}\right)+\left(k_{B} T\right)^{2} \frac{\pi \Gamma E}{3\left(\Gamma^{2}+E^{2}\right)^{2}} .
\end{aligned}
$$

If the temperatures are high $\left(T>T_{\Gamma, E}\right)$ then

$$
\begin{aligned}
& \mathscr{g}_{D}^{\prime}(E, \Gamma) \approx \ln \left(\frac{1.13 \pi T_{D}}{T}\right)+O\left(\frac{T_{\Gamma, E}}{T}\right), \\
& \hat{f}(E, \Gamma) \approx \frac{1}{2}-\left(\frac{0.6433 \beta E}{2 \pi}+\frac{0.426 \beta^{2} \Gamma E}{\pi}\right) .
\end{aligned}
$$

Analytic expressions for the intermediate range can also be determined, but it becomes simpler just to evaluate $\psi\left(\frac{1}{2}+z\right)$ on a computer and solve with self-consistency at the same time. An algorithm for $\psi\left(\frac{1}{2}+z\right)$ can be easily constructed from a code for the gamma function in the complex plane. ${ }^{90}$

\section{APPENDIX D: COMPARISON OF THE ATOMIC LIMIT GREEN'S FUNCTION IN THE EXACT AND HF FORMS}

In this appendix, we show the relationship between the one-electron Green's function in the atomic limit and the Hartree-Fock approximation thereto. We wish to show that in the grand canonical ensemble the Hartree-Fock energy is a particularly poor estimator of the existence of an interconfigurational excitation energy (ICEE) near the Fermi energy (i.e., near zero).

The demonstration begins by writing the oneelectron quantum numbers as $\alpha$ and expanding $c_{\alpha}$ :

$$
c_{\alpha}=\sum_{n, \gamma_{n}, \gamma_{n+1}} a_{\gamma_{n}, \gamma_{n+1}}^{\alpha} \tilde{\Phi}\left(\gamma_{n}, \gamma_{n+1}\right)
$$

where $\tilde{\Phi}$ is the non-normalized Judd stepping operator [cf. Eqs. (3.10) and (3.11), for example]

$$
\tilde{\Phi}\left(\gamma_{n}, \gamma_{n+1}\right)=A_{\gamma_{n}}^{\dagger} I_{\theta} A_{\gamma_{n+1}},
$$

between configurations $\gamma_{n}$ and $\gamma_{n+1}$ which are created from the vacuum by the operators $A_{\gamma_{n}}^{\dagger}$ and $A_{\gamma_{n+1}}^{\dagger}$, respectively. The $a_{\gamma, \gamma}$ coefficients are closely related in spherical symmetry to the Clebsch-Gordan coefficients, but their values are not of importance at this point. By straightforward calculation it can be shown that

$$
\left(\left(\Phi\left(\gamma_{n}, \gamma_{n+1}\right), \Phi\left(\gamma_{n}, \gamma_{n+1}\right)\right)\right)=P_{\gamma_{n}}+P_{\gamma_{n+1}},
$$

where $P_{\gamma_{n}}$ is the probability at temperature $T$ and chemical potential $\mu$ (in the grand cononical ensemble) that $n$-electron state $\gamma_{n}$ is occupied. The atomic-limit impurity Green's function is then given by

$$
\left(\left(c_{\alpha},(\omega-L)^{-1} c_{\alpha}\right)\right)=\sum_{n, \gamma_{n}, \gamma_{n+1}} \frac{\left|a_{\gamma_{n}, \gamma_{n+1}}^{\alpha}\right|^{2}\left(P_{\gamma_{n}}+P_{\gamma_{n+1}}\right)}{\omega-\left(E_{\gamma_{n+1}}-E_{\gamma_{n}}-\mu\right)},
$$

where the energies of the $n$-electron atomic eigenstates $\gamma_{n}$ have been written as $E_{\gamma_{n}}$.

Now it is a property of the Hartree-Fock approximation that it preserves the first moment of the one electron Green's function

$\left(\left(c_{\alpha},(\omega-L)^{-1} c_{\alpha}\right)\right)=\frac{1}{\omega}+\frac{1}{\omega^{2}}\left(\left(c_{\alpha}, L c_{\alpha}\right)\right)+\cdots$.

This can be verified by recalling that the substance of the Hartree-Fock approximation is to find the matrix elements of $L$ completely within the oneelectron subspace, or equivalently to determine the effect of $L$ only on the $c_{\alpha}$. This yields

$$
L c_{\alpha}=\left(\left(c_{\alpha}, L c_{\alpha}\right)\right) c_{\alpha}+\cdots,
$$

where the neglected terms involve more than one $c_{\alpha}$.

To complete the demonstration we examine the first moment of the exact Green's function,

$$
\begin{gathered}
\left(\left(c_{\alpha}, L c_{\alpha}\right)\right)=\sum_{n, \gamma_{n}, \gamma_{n+1}}\left|a_{\gamma_{n}, \gamma_{n+1}}^{\alpha}\right|^{2}\left(P_{\gamma_{n}}+P_{\gamma_{n+1}}\right) \\
\times\left(E_{\gamma_{n+1}}-E_{\gamma_{n}}-\mu\right),
\end{gathered}
$$

and observe that if there is an ICEE which is very small, its contribution could easily be dominated by other transitions from the ground state of the atom. Therefore, Hartree-Fock calculations should not always be trusted as an indicator of many electron ICEE's close to the Fermi energy.
${ }^{1}$ P. W. Anderson, Phys. Rev. 124, 41 (1961).

${ }^{2}$ J. R. Schrieffer, J. Appl. Phys. 38, 1143 (1967).

${ }^{3}$ L. L. Hirst, Kondens. Mater. 11, 255 (1970); J. Phys. Chem. Solids 35, 1285 (1974); J. Appl. Phys. 39, 849 (1968).

${ }^{4}$ The operators used are elements of the Lie algebra associated with the $S$ shell of the impurity and the conduction electrons. See B. R. Judd, Topics in Atomic and Nuclear Theory (University of Canterbury, New Zealand, 1970), p. 21.

${ }^{5}$ E. Feenberg, Phys. Rev. 74, 206 (1948).

${ }^{6}$ S. P. Bowen, J. Math. Phys. 16, 620 (1975).

${ }^{7} \mathrm{~J}$. Kondo, Prog. Theor. Phys. 32, 37 (1964).

${ }^{8} \mathrm{~J}$. Friedel, Nuovo Cimento Suppl. 1, 287 (1958). 
${ }^{9}$ J. Kondo, Solid State Physics, edited by F. Seitz, D. Turnbull and H. Ehrenreich (Academic, New York, 1960), Vol. 23, p. 183; C. Rizzuto, Rep. Prog. Phys. 37,147 (1974).

${ }^{10}$ G. Bruner, Adv. Phys. 23, 941 (1974).

${ }^{11} \mathrm{P}$. W. Anderson and W. L. McMillan, in Theory of Magnetism in Transition Metals, Varenna, 1966, edited by W. Marshall (Academic, London, 1967); see also Table I in Ref. 10.

${ }^{12}$ See Ref. 3.

${ }^{13}$ See Ref. 1.

${ }^{14}$ H. Suhl and D. R. Fredkin, Phys. Rev. 131, 1063 (1963); A. C. Hewson and M. J. Zuckermann, Phys. Lett. 20, 219 (1966).

${ }^{15}$ See Ref. 9.

${ }^{16}$ C. S. Stassis and C. G. Shull, Phys. Rev. B 5,1040 (1972); N. Kroo and Z. Szentirmay, Phys. Lett. A $\underline{40}$, 173 (1972)

${ }^{17}$ K. G. Wilson, Nobel Symp. 24, 68 (1973).

${ }^{18}$ H. R. Krishnamurti, K. G. Wils on, and J. W. Wilkins, Phys. Rev. Lett. 35, 1101 (1975).

${ }^{19}$ D. Masson, "Hilbert Space and the Pade Approximant," in The Pade Approximant in Theoretical Physics, edited by G. A. Baker, Jr. and John L. Gammel (Academic, New York, 1970), p. 197ff.

${ }^{20}$ Situations where degeneracy breaking leads to large effects from small interactions are widespread, superconductivity and charge-density waves being two examples.

${ }^{21}$ Examination of ICEE estimates for Mn from C. Moores [Atomic Energy Levels as Derived from the Analysis of Optical Spectra, Natl. Bur. Stds. Circ. No. 467 (U. S. GPO, Washington, D. C., 1949)] gives values in the range $5-7 \mathrm{eV}$.

${ }^{22}$ J. J. Peters and C. P. Flynn, Phys. Rev. B 6,3343 (1972).

${ }^{23}$ See Ref. 3

${ }^{24}$ For most of the remainder of the article the one-electron energies $\epsilon_{d s}$, etc., will be measured with respect to the Fermi energy.

${ }^{25}$ M. B. Maple and D. K. Wohlleben, AIP Conf. Proc. 18, 447 (1973).

${ }^{26}$ See Ref. 3.

${ }^{27}$ See Ref. 2.

${ }^{28} \mathrm{~J}$. C. Slater, Quantum Theory of Atomic Structure (McGraw-Hill, New York, 1960), Vol. II; B. R. Judd, operator Techniques in Atomic Spectroscopy (McGraw-Hill, New York, 1967).

${ }^{29}$ See Ref. 3.

${ }^{30}$ As noted in Ref. 24 , the conduction-electron energies $\epsilon_{\overrightarrow{\mathrm{k}} s}$ are measured with respect to the Fermi energy, i.e., the chemic al potential has already been subtracted off.

${ }^{31} \mathrm{C}$. Herring, Magnetism IV, edited by G. T. Rado and H. Suhl (Ac ademic, New York, 1966).

${ }^{32}$ S. P. Bowen, J. Math. Phys. 16, 620 (1975).

${ }^{33} \mathrm{D}$. K. Wohlleben and B. R. Coles, in Magnetism V, edited by G. T. Rado and H. Suhl (Academic, New York, 1973), p. 3.

${ }^{34}$ See Ref. 14

${ }^{35}$ See Ref. 1.

${ }^{36}$ J. Hubbard, Proc. R. Soc. A 277, 237 (1963).

${ }^{37}$ E. Feenberg, Phys. Rev. 74, 206 (1948); S. P. Bowen, J. Math. Phys. 16, 620 (1975).

${ }^{38}$ The unit-step function is +1 for $t>0$ and +0 for $t<0$.

${ }^{39}$ J. Hubbard, Proc. R. Soc. A 276, 238 (1963).
${ }^{40}$ D. Zubarev, Usp. Fiz. Nauk 71, 71 (1960) [Sov. Phys.Usp. 3 , 320 (1960)].

${ }^{41}$ See Ref. 32 .

${ }^{42}$ A. Lonke, J. Math. Phys. 12, 2422 (1971).

${ }^{43}$ See Ref. 37.

${ }^{44}$ See 'Refs. 24 and 30.

${ }^{45}$ See Ref. 42.

${ }^{46}$ See (a) B. R. Judd, Topics in Atomic and Nuclear Theory (University of Canterbury, New Zealand, 1970), Chap. V; (b) Ref. 28 (Judd, Chap. 5).

${ }^{47}$ See Ref. 46 (a) (p. 22); also F.A.Berezin, The Method of Second Quantization (Ac ademic, New York, 1966), p. $28 \mathrm{ff}$.

${ }^{48}$ See Ref. 46 (a).

${ }^{49}$ L. P. Kadanoff and G. Baym, Quantum Statistical Mechanics (Benjamin, New York, 1962).

${ }^{50}$ See Ref. 2.

${ }^{51}$ See Ref. 42 .

${ }^{52}$ L. F. Bates and M. M. Newmann, Proc. Phys. Soc. London 72,345 (1958)

${ }^{53}$ K. S. Kim and M. B. Maple, Phys. Rev. B 2, 4696 (1970); W. Gey and E. Umlauf, Z. Phys. 242, 241 (1971).

${ }^{54}$ See first of Ref. 53.

${ }^{55}$ See Ref. 53.

${ }^{56}$ See Ref. 32 .

${ }^{57} \mathrm{~J}$. C. Slater, Quantum Theory of Atomic Structure. (Mc Graw-Hill, New York, 1960), Vol. I.

${ }^{58}$ In the thermodynamic limit the contribution to the self-energy from a single conduction-electron state is of measure zero and so countably many of these terms $c$ an be omitted or included with no effect.

${ }^{59} \mathrm{~A}$. Percheron et al., Solid State Commun. 12, 1289 (1973); K. Andres and J. E. Graebner, Phys. Rev. Lett. 35, 1779 (1975); B. Cornut and B. Coqblin, Phys. Rev. B 5, 4541 (1972).

${ }^{60}$ See Ref. 9.

${ }^{61}$ C. 'Rizzuto, Rep. Prog. Phys. 37, 147 (1974).

${ }^{62} \mathrm{~J}$. W. Loram, T. E. Whall, and P. J. Ford, Phys. Rev. B 2, 857 (1970).

${ }^{63}$ P. E. Bloomfield and D. R. Hamann, Phys. Rev. Lett. 164, 856 (1967).

${ }^{64}$ See Ref. 61.

${ }^{65}$ P. Monod, Phys. Rev. Lett. 19, 1113 (1967).

${ }^{66}$ M. D. Daybell and W. A. Steyert, Phys. Rev. Lett. 20, 195 (1968).

${ }^{67}$ D. K. C. McDonald, Thermoelectricity: An Introduction to the Principles (Wiley, New York, 1962).

${ }^{68}$ See Ref. 61, p. 219.

${ }^{69}$ See Ref. 22.

${ }^{70}$ See Ref. 33.

${ }^{71}$ N. Rivier and M. J. Zuckermann, Phys. Rev. Lett. 21, 904 (1968).

${ }^{72} \mathrm{~J}$. H. Van Vleck, The Theory of Electric and Magnetic Susceptibilities (Oxford University, London, 1932).

${ }^{73}$ See Ref. 61, p. 158.

${ }^{74}$ E. C. Hirschkoff, O. G. Symko, and G. C. Whe atley, J. Low Temp. Phys. $\underline{5}, 155$ (1971).

${ }^{75}$ See Ref. 10.

${ }^{76}$ D. J. Scalapino, Phys. Rev. Lett. 16, 937 (1966).

${ }^{77}$ S. D. Bader, N. E. Phillips, M. B. Maple, and C. A. Luengo (unpublished).

${ }^{78}$ M. Gomez, S. P. Bowen, and J. A. Krumhansl, Phys. Rev. 153, 1009 (1967).

${ }^{79}$ See Ref. 17. 
${ }^{80}$ See Ref. 18.

${ }^{81}$ S. P. Bowen (unpublished).

${ }^{82}$ See Slater in Ref. 28.

${ }^{83}$ See Judd in Ref. 28.

${ }^{84}$ See Ref. 61.

${ }^{85}$ See Ref. 61 .

${ }^{86}$ See Ref. 10 .

${ }^{87}$ See Ref. 2.

${ }^{88}$ I. S. Gradshteyn and I. M. Rhyzik, Table of Integrals,
Series and Products (Academic, New York, 1965), p. 328.

${ }^{89}$ Philip J. Davis, in Handbook of Mathematical Functions, edited by M. Abramowitz and I. A. Stegun

(Dover, New York, 1965), p. 259.

${ }^{90}$ A. K. Hershey, Approximations of Functions by Sets of Poles, NWL Technical Report RT-2564 (U.S.

Naval Weapons Laboratory, Dahlgren, Va. 22448, 1971). 